\title{
Where do we stand with solar neutrino oscillations?
}

\author{
J. N. Bahcall \\ School of Natural Sciences, Institute for Advanced Study \\ Princeton, NJ 08540 \\ P. I. Krastev \\ University of Wisconsin, Madison, WI 53706
}

A. Yu. Smirnov

ICTP, Trieste, Italy

\begin{abstract}
We determine parameters for MSW and vacuum oscillations (active and sterile neutrinos) that are allowed by separate, and collective, imposition of the constraints from total event rates in the chlorine, GALLEX, SAGE, and SuperKamiokande experiments (504 days), the SuperKamiokande energy spectrum, and the SuperKamiokande zenith-angle dependence. The small mixing angle MSW solution is acceptable at 7\% C.L. (8\% for sterile $\nu$ 's) and the vacuum solution is acceptable at $6 \%$ C.L. . The best-fit global MSW solution for active neutrinos is: $\Delta m^{2}=5 \times 10^{-6} \mathrm{eV}^{2}, \sin ^{2} 2 \theta=5.5 \times 10^{-3}$ (and for sterile neutrinos: $\left.\Delta m^{2}=4 \times 10^{-6} \mathrm{eV}^{2}, \sin ^{2} 2 \theta=7 \times 10^{-3}\right)$. For vacuum oscillations, the best-fit solution is: $\Delta m^{2}=6.5 \times 10^{-11} \mathrm{eV}^{2}, \sin ^{2} 2 \theta=0.75$. An arbitrary combination of undistorted (no oscillations) $p p,{ }^{7} \mathrm{Be},{ }^{8} \mathrm{~B}$, and $\mathrm{CNO}$ neutrino fluxes is inconsistent with the combined data sets at the $3.5 \sigma$ C.L., independent of astrophysical considerations. We use improved calculations of solar model fluxes, neutrino absorption cross sections and energy spectra, and a detailed evaluation of regeneration effects.
\end{abstract}

26.65.+t, 12.15.Ff, 14.60.Pq, 13.15.+g, 96.60.Jw 


\section{INTRODUCTION}

It is now 30 years since the first recognition of the solar neutrino problem [1 3]. In the first two decades of solar neutrino research [⿴囗⿴囗十), the problem consisted only of the discrepancy between theoretical calculations based upon on a standard solar model (with the implicit assumption that neutrinos created in the solar interior reach the earth unchanged) and the observations of the capture rate in the chlorine solar neutrino experiment.

In recent years, four new experiments (Kamiokande [5], GALLEX [6], SAGE [7], and SuperKamiokande [8 10]) have reported results. All four experiments confirm the original detection of solar neutrinos with lower neutrino fluxes than predicted by standard solar models. In addition, the Kamiokande and SuperKamiokande experiments demonstrate directly that the neutrinos come from the sun by showing that recoil electrons are scattered in the direction along the sun-earth axis.

In April 1996, the SuperKamiokande experiment initiated a new era of high-precision and high-statistics solar neutrino research. The first 504 days of data from SuperKamiokande [10], when combined with data from earlier experiments on solar neutrinos, provide important constraints on the MSW [11] and vacuum oscillation [12] solutions of the solar neutrino problem.

The definitive analysis of the implications of the SuperKamiokande data set must await the comprehensive Monte Carlo study that can only be performed by the SuperKamiokande Collaboration. However, the excellent agreement of the results from the first 300 days of SuperKamiokande operation [8] with the results obtained after 374 days [9] and after 504 days [10] shows the robustness of the results. Therefore, with more than a year's worth of data available, this is an appropriate time to take stock of what has been achieved and what further challenges lie ahead. The analysis presented here will, we hope, be useful in guiding experimental plans for other detectors such as SNO [13] and BOREXINO [14]. We also hope that our summaries of the analysis techniques and the theoretical input data (see especially Sec. III and the Appendix), as well as the indicated results, will be helpful to others who will make similar studies. The results from different theoretical analyses should be compared with each other, and with the comprehensive studies by the SuperKamiokande Collaboration using their detailed Monte Carlo simulation, in order to test the robustness of the inferences about neutrino parameters.

In this paper, we explore the implications of the 504 day data set from SuperKamiokande together with the results from the chlorine, GALLEX, and SAGE experiments. For a concise summary of our conclusions, the reader is advised to skip directly to Sec. VIII and then to return to this introduction.

We use improved neutrino interaction cross sections [15 18, the most accurate neutrino spectra [15,19], and the results of a recent reevaluation, the BP98 model, by Bahcall and Pinsonneault of the standard model neutrino fluxes [20]. The BP98 model is based upon a comprehensive examination of all of the available nuclear fusion data 21] that was carried out under the auspices of the Institute of Nuclear Theory (INT). For solar neutrino research, the most important nuclear physics parameter is the low energy cross section factor, $S_{17}$, for the reaction ${ }^{7} \mathrm{Be}(p, \gamma){ }^{8} \mathrm{~B}$, which gives rise to the critical ${ }^{8} \mathrm{~B}$ neutrinos. The INT normalization, $S_{17}(\mathrm{INT})=19_{-2}^{+4} \mathrm{eV}$ b [21], is about $1 \sigma$ less than the previously standard Caltech (CIT) cross section factor [22], $S_{17}(\mathrm{CIT})=(22.4 \pm 2.1) \mathrm{eV}$ b. The CIT normalization was computed 
by taking the weighted average over all the published experimental data while the INT normalization was computed by including only the results from the two most recent and best documented experiments. Because the uncertainty in the ${ }^{8} \mathrm{~B}$ production cross section is the most important uncertainty in predicting solar neutrino fluxes [20], we present results in this paper for both the INT and the CIT normalizations of $S_{17}$.

We also present calculations for oscillations into sterile neutrinos as well as into the more familiar active neutrinos. In what follows, we shall always mean oscillations into active neutrinos unless we explicitly include the adjective 'sterile.' We do not consider here the intermediate case of oscillations partly into active neutrinos and partly into sterile neutrinos, although this is a logical possibility. For plausible assumptions (see e.g. [23]), consistent with the results of the CHOOZ experiment [24], the oscillations of solar neutrinos are well described by the two-generation formalism. Therefore, we consider explicitly here only two neutrino generations.

We begin by considering in Sec. [1 how well (or rather how poorly) the results of solar neutrino experiments are described by the combined predictions of the standard solar model and the minimal electroweak theory (which implies that nothing happens to the neutrinos after they are created). In Sec. III we briefly summarize the ingredients and techniques used in our analysis and give references to the original sources for the improved neutrino flux calculations, the associated uncertainties in the fluxes, the improved neutrino energy spectra and interaction cross sections, the methods including theoretical errors, and the techniques for carrying out the theoretical calculations. Details of the statistical analysis are provided in the Appendix. We determine in Sec. $\mathbb{V}$ the regions that are allowed in neutrino parameter space for MSW and vacuum oscillations to either active or sterile neutrinos provided only the total event rates in the neutrino experiments are considered. In Sec. $\nabla$ we determine the implications of the zenith-angle dependence of the SuperKamiokande event rates. We quantify in Sec. VI the distortion of the recoil electron energy spectrum measured by SuperKamiokande, determining the slope parameter and the excluded regions of oscillation parameters. We impose in Sec. VIT all of the constraints, total rates, electron recoil energy spectrum, and zenith-angle dependence, in global fits and determine the range of oscillation parameters that are consistent with all the data. We present in Sec. VIII our summary and overview of where we stand in understand the discrepancies between standard model predictions and the results of solar neutrino experiments and in the determination of neutrino parameters from solar neutrino experiments.

\section{WHAT IS ALL THE FUSS ABOUT?}

Why are so many papers being written about non-standard physics implied by solar neutrino experiments? This section provides two answers to this question. In Sec. IIA, we show that all of the 19 standard solar model calculations published in refereed journals in the last 10 years predict neutrino fluxes that are in reasonable agreement with each other.

In Sec. [IB, we show that the measured rates and their uncertainties in solar neutrino experiments are inconsistent with any combination of the solar neutrino fluxes that does not include a spectrum distortion-which requires physics beyond the standard electroweak model. We summarize in Sec. IIC the comparisons between the predictions of the standard model-minimal electroweak theory plus standard solar model-and the results of solar 
neutrino experiments.

\section{A. The last decade of standard solar models}

Figure 1 displays the calculated ${ }^{7} \mathrm{Be}$ and ${ }^{8} \mathrm{~B}$ neutrino fluxes for all 19 standard solar models with which we are familiar which have been published in the last 10 years in refereed science journals. The fluxes are normalized by dividing each published value by the flux from the BP98 solar model [20]; the abscissa is the normalized ${ }^{8} \mathrm{~B}$ flux and the ordinate is the normalized ${ }^{7} \mathrm{Be}$ neutrino flux. The rectangular box shows the estimated $3 \sigma$ uncertainties in the predictions of the BP98 solar model. The abbreviations, which indicate references to individual models, are identified in the caption of Figure 1.

All of the solar model results from different groups fall within the estimated $3 \sigma$ uncertainties in the model predictions (with the exception of the Dar-Shaviv model whose results have not been reproduced by other groups). This agreement demonstrates the robustness of the predictions since the calculations use different computer codes (which achieve varying degrees of precision) and involve a variety of choices for the nuclear parameters, the equation of state, the stellar radiative opacity, the initial heavy element abundances, and the physical processes that are included.

The largest contributions to the dispersion in values in Figure 1 are due to the choice of the normalization for $S_{17}$ (the production cross-section factor for ${ }^{8} \mathrm{~B}$ neutrinos) and the inclusion, or non-inclusion, of element diffusion in the stellar evolution codes. The effect in the plane of Fig. 1 of the normalization of $S_{17}$ is shown by the difference between the point for BP98 (1.0,1.0), which was computed using the INT normalization, and the point at $(1.18,1.0)$ which corresponds to the BP98 result with the CIT normalization.

Helioseismological observations have shown recently 25] that diffusion is occurring and must be included in solar models, so that the most recent models shown in Fig. 1 now all include helium and heavy element diffusion. By comparing a large number of earlier models, it was shown that all published standard solar models give the same results for solar neutrino fluxes to an accuracy of better than $10 \%$ if the same input parameters and physical processes are included [26,27].

How do the observations from the solar neutrino experiments agree with the solar model calculation?

Table 1 summarizes the solar neutrino experimental rates that have been measured in the five experiments. We have compared the observed rates with the calculated, standard model values, combining quadratically the theoretical solar model and experimental uncertainties, as well as the uncertainties in the neutrino cross sections. Since the GALLEX and SAGE experiments measure the same quantity, we treat the weighted average rate in gallium as one experimental number. We adopt the SuperKamiokande measurement as the most precise direct determination of the higher-energy ${ }^{8} \mathrm{~B}$ neutrino flux.

Using the predicted fluxes from the BP98 model, the $\chi^{2}$ for the fit to the three experimental rates (chlorine, gallium, and SuperKamiokande) is

$$
\chi_{\mathrm{SSM}}^{2}(3 \text { experimental rates })=61 \text {. }
$$

The result given in Eq. (11), which is approximately equivalent to a $20 \sigma$ discrepancy, is 
a quantitative expression of the fact that the standard model predictions do not fit the observed solar neutrino measurements.

\section{B. Model-independent tests}

Suppose (following the precepts of Hata et al. [28], Parke [29], and Heeger and Robertson [30]) we now ignore everything we have learned about solar models over the last 35 years and allow the important $p p,{ }^{7} \mathrm{Be}$, and ${ }^{8} \mathrm{~B}$ fluxes to take on any non-negative values. What is the minimum value of $\chi^{2}$ for the 3 experiments, when the only constraint on the fluxes is the requirement that the luminosity of the sun be supplied by nuclear fusion reactions among light elements? We include the nuclear physics inequalities between neutrino fluxes (see section 4 of Ref. [31]) that are associated with the luminosity constraint and maintain the standard value for the essentially model-independent ratio of $p e p$ to $p p$ neutrino fluxes.

\section{With SSM value for CNO neutrinos}

We begin by allowing the $p p,{ }^{7} \mathrm{Be}$, and ${ }^{8} \mathrm{~B}$ neutrino fluxes to be arbitrary parameters (subject to the luminosity constraint), but constrain the ${ }^{13} \mathrm{~N}$ and ${ }^{15} \mathrm{O}$ fluxes to be equal to the values predicted by the standard solar model [20]. There is therefore one degree of freedom for three experiments plus the luminosity constraint and three freely chosen neutrino fluxes. The best fit for arbitrary $p p,{ }^{7} \mathrm{Be}$, and ${ }^{8} \mathrm{~B}$ fluxes is obtained for ${ }^{7} \mathrm{Be} /\left({ }^{7} \mathrm{Be}\right)_{\mathrm{SSM}}=0.0$, ${ }^{8} \mathrm{~B} /\left({ }^{8} \mathrm{~B}\right)_{\mathrm{SSM}}=0.44$, and $p p=1.08$, where

$$
\chi_{\text {minimum }}^{2}\left(3 \text { experimental rates; arbitrary } p p,{ }^{7} \mathrm{Be},{ }^{8} \mathrm{~B}\right)=24.1 \text {. }
$$

The best-fit solution differ considerably from the measured values for the radiochemical experiments, with 3.4 SNU for chlorine and 95.5 SNU for gallium, but is in good agreement with the measured value (0.474 of BP98) for SuperKamiokande (cf. Table I).

There are no acceptable fits at a C.L. of more than $99.99 \%$ ( $4 \sigma$ result).

\section{CNO neutrinos assumed missing}

The fit can be improved if we set the CNO neutrino fluxes equal to zero. fo Then, the same search for arbitrary $p p,{ }^{7} \mathrm{Be}$, and ${ }^{8} \mathrm{~B}$ neutrino fluxes leads to a best fit with ${ }^{7} \mathrm{Be} /\left({ }^{7} \mathrm{Be}\right)_{\mathrm{SSM}}=$ $0.0,{ }^{8} \mathrm{~B} /\left({ }^{8} \mathrm{~B}\right)_{\mathrm{SSM}}=0.46$, and $p p /(p p)_{\mathrm{SSM}}=1.10 .$. The minimum value of $\chi^{2}$ is

$$
\chi_{\text {minimum }}^{2}\left(3 \text { experimental data; arbitrary } p p,{ }^{7} \mathrm{Be},{ }^{8} \mathrm{~B} ; \mathrm{CNO}=0\right)=7.3 \text {. }
$$

The best-fit solution has 2.9 SNU for chlorine and 85.3 SNU for gallium, and 0.46 of BP98 for SuperKamiokande (cf. Table [). Although these three best-fit values are not far from the

\footnotetext{
*If the CNO neutrino fluxes are allowed to vary as free parameters, the minimum $\chi^{2}$ is achieved for zero CNO fluxes.
} 
three measured values, the best-fit values were found by an extensive computer search with three free parameters and only one physical constraint, that the nuclear energy generation rate correspond to the total luminosity of the sun.

There are no acceptable solutions at the $99 \%$ C. L. ( $\sim 3 \sigma$ result $)$.

Figure 1 shows the best-fit solution and the $1 \sigma-3 \sigma$ contours. The $1 \sigma$ and $3 \sigma$ limits were obtained by requiring that $\chi^{2}=\chi_{\min }^{2}+\delta \chi^{2}$, where for $1 \sigma \delta \chi^{2}=1$ and for $3 \sigma \delta \chi^{2}=9$. All of the standard model solutions lie far from the best-fit solution and even lie far from the $3 \sigma$ contour.

\section{Discussion of comparisons with standard model}

The searches for best-fit solutions that are described by Eq. (2) and Eq. (3) have three independent experimental data points and 1 additional constraint (the luminosity constraint) with three free parameters (the $p p,{ }^{7} \mathrm{Be}$, and ${ }^{8} \mathrm{~B}$ neutrino fluxes). We conclude from Eq. (21) and Eq. (3) that all solutions with undistorted energy spectra are ruled out at the $99 \%$ C.L. (with the CNO neutrino fluxes set equal to zero, see Eq. (31) or at more than 99.9\% C.L. (with the CNO fluxes equal to the values predicted by the standard solar model).

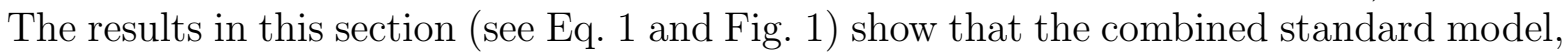
the standard solar model plus minimal standard electroweak theory, provides a bad fit to the observed rates in solar neutrino experiments. Moreover, Eq. (2) and Eq. (3) show that one cannot get a good fit to the observed rates using any combination of undistorted neutrino energy spectra. All so-called astrophysical solutions give a poor description of the observed experimental rates. This is what all the fuss is about.

If we drop the physical requirement that the fluxes be positive definite, the minimum $\chi^{2}\left(\chi_{\min }^{2}=0.1\right)$ occurs-quite remarkably- for a negative value of the ${ }^{7} \mathrm{Be}$ flux; $\phi\left({ }^{7} \mathrm{Be}\right)_{\min }=$ $-0.45 \times \phi\left({ }^{7} \mathrm{Be}\right)_{\mathrm{SSM}}$. This unphysical result is a reflection of what has become known as the problem of " the missing ${ }^{7} \mathrm{Be}$ solar neutrinos.". One reason that the ${ }^{7} \mathrm{Be}$ neutrinos appear to be missing (or have a negative flux) is that the two gallium experiments, GALLEX and SAGE, have an average event rate of $72.3 \pm 5.6 \mathrm{SNU}$, which is fully accounted for in the standard solar model by the fundamental $p p$ and pep neutrinos (72.4 SNU) [26,27.20]. In addition, the ${ }^{8} \mathrm{~B}$ neutrinos that are observed in the Kamiokande and SuperKamiokande experiments will produce about $6 \mathrm{SNU}$ in the gallium experiments, unless new particle physics changes the neutrino energy spectrum. A second reason that the ${ }^{7} \mathrm{Be}$ flux appears to be missing is that (see [32]) the SuperKamiokande ${ }^{8} \mathrm{~B}$ neutrino flux alone corresponds to $2.70(1 \pm 0.11) \mathrm{SNU}$ in a chlorine detector (combining quadratically the theoretical cross section errors with the SuperKamiokande measurement errors), which is to be compared to the total observed rate in the Homestake chlorine experiment of $(2.56 \pm 0.23) \mathrm{SNU}$. The observed rate includes neutrinos from ${ }^{8} \mathrm{~B},{ }^{7} \mathrm{Be}, \mathrm{CNO}$, and pep. Obviously, there is no room in the measured chlorine rate for a significant ${ }^{7} \mathrm{Be}$ neutrino contribution (expected to be 1.15 SNU based upon the standard solar model [20]).

For all these reasons, we will consider in the remainder of this paper theories in which neutrino oscillations change the shape of the neutrino energy spectra. 


\section{INGREDIENTS AND TECHNIQUES}

Many authors have reported the results of refined studies of the MSW [33 41] and the vacuum [37,38, 40,42] solutions of the solar neutrino problems. The techniques for this analysis are therefore well documented in the literature and we only note briefly here those aspects of the calculation that are often not treated in the optimal manner in the literature or for which less accurate data are sometimes used. Our $\chi^{2}$ analysis of the data follows closely the prescriptions in [43,31]. We adopt the procedures of Fogli and Lisi (see Ref. [34]) in including theoretical errors. The uncertainties in the input model parameters that influence the neutrino fluxes are taken from Refs. [20,27]. We use the improved neutrino interaction cross sections for each detector given in Refs. 15 18 and the neutrino spectra given in Refs. [15,19]. We include the published energy resolution and trigger efficiency of the Kamiokande detector [5] and SuperKamiokande [8-10]. For the MSW solution, we use the analytical description of the neutrino survival probabilities from 44 which allows the averaging over the neutrino production regions and the neutrino spectra to be done accurately with a reasonable amount of computer time.

We obtain allowed regions in $\Delta m^{2}-\sin ^{2} 2 \theta$ parameter space by finding the minimum $\chi^{2}$ and plotting contours of constant $\chi^{2}=\chi_{\min }^{2}+\Delta \chi^{2}$ where $\Delta \chi^{2}=5.99$ for $95 \%$ C.L. and 9.21 for $99 \%$ C.L. . In all the figures in this paper, we show results at the $99 \%$ C.L.

We describe the statistical analysis in detail in the Appendix.

\section{FITS TO THE AVERAGE EVENT RATES}

In this section, we determine the allowed range of oscillation solutions using only the total event rates in the ClAr (Homestake), GALLEX, SAGE, and SuperKamiokande experiments.]

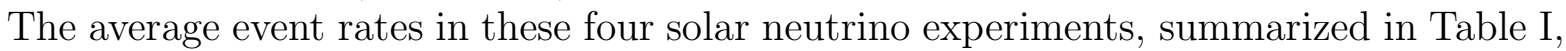
are robust and seem unlikely to change significantly. The results of the chlorine experiment have been summarized in detail recently [45; the measured value has been stable for two decades and is known relatively precisely. The Kamiokande [5] and SuperKamiokande [8 10] experiments are in agreement to within $1 \sigma$ and the precision of the SuperKamiokande experiment is now very high. We have used the more precise value of the SuperKamiokande experiment to represent the total rate above $6.5 \mathrm{MeV}$ in the water Cherenkov experiments. Both the GALLEX [6] and the SAGE [0] experiments have yielded accurate measurements and the efficiency of both detectors has been tested with ${ }^{51} \mathrm{Cr}$ sources. We have used the weighted average of the measured rates in the two gallium detectors.

We present in Sec. IVA the allowed range of solutions involving MSW oscillation into active or sterile neutrinos. In Sec. IVB, we present the corresponding results for vacuum oscillations. Sec. IVC describes the dependence of the inferred oscillation parameters on

\footnotetext{
†We have carried out identical calculations including both the Kamiokande and the SuperKamiokande rates. The results are essentially unchanged from what we find including only the SuperKamiokande rate, since the quoted uncertainty in the Kamiokande rate is much larger than the uncertainty in the SuperKamiokande rate.
} 
the most uncertain input parameter, the low-energy cross section factor for the ${ }^{7} \operatorname{Be}(p, \gamma)^{8} \mathrm{~B}$ reaction. We show in Sec. IVD that energy-independent oscillations are unacceptable at the 99.8\% C.L. . Finally, we summarize in Sec. IVE our results on the analysis of the average event rates.

The calculation of the allowed range of predicted ${ }^{7}$ Be flux (to be measured in the BOREXINO experiment [14]), the demonstration that a constant suppression factor is a disfavored description, and the evaluation of the dependence of the inferred neutrino parameters on the low-energy cross section factor for the ${ }^{7} \mathrm{Be}(p, \gamma)^{8} \mathrm{~B}$ reaction are special features of this section.

\section{A. MSW solutions}

\section{Active neutrinos}

The best fit is obtained for the small mixing angle (SMA) solution:

$$
\begin{aligned}
\Delta m^{2} & =5.4 \times 10^{-6} \mathrm{eV}^{2}, \\
\sin ^{2} 2 \theta & =6.0 \times 10^{-3},
\end{aligned}
$$

which has $\chi_{\min }^{2}=1.7$. There are two more local minima of $\chi^{2}$. The best fit for the well known large mixing angle (LMA) solution occurs at

$$
\begin{aligned}
\Delta m^{2} & =1.8 \times 10^{-5} \mathrm{eV}^{2}, \\
\sin ^{2} 2 \theta & =0.76,
\end{aligned}
$$

with $\chi_{\min }^{2}=4.3$. There is also a less probable solution, which we refer to as the LOW solution (low probability, low mass), at 46,47

$$
\begin{aligned}
\Delta m^{2} & =7.9 \times 10^{-8} \mathrm{eV}^{2}, \\
\sin ^{2} 2 \theta & =0.96 .
\end{aligned}
$$

with $\chi_{\min }^{2}=7.3$. The LOW solution is acceptable at the $99 \%$ C.L., but is not acceptable at the $95 \%$ C. L. . To find an appreciable probability for the LOW solution, one must include the regeneration effect 43].

How do the results given in Eq. (四)-Eq. (6) differ from our pre-SuperKamiokande (1997) analysis? (The 1997 study, Ref. [43], was carried out before either the Bahcall-Pinsonneault 98 solar model or the SuperKamiokande experimental results were available.) There are no very large changes in the best-fit values for either the mass differences or the mixing angles of the three solutions, although the $\chi^{2}$ fits are less good (but well within the statistical uncertainties, cf. Eqs. (1)-(3) of Ref. [43]). However, the best-fit neutrino parameters are shifted towards smaller mixing angles for the SMA and towards larger mixing angles for the LMA and LOW solutions. For the SMA solution, this has the important result that the expected Day-Night asymmetry is greatly decreased (see Sec. V.)

Figure 2 shows the $99 \%$ C.L. allowed regions in the plane defined by $\Delta m^{2}$ and $\sin ^{2} 2 \theta$. The black dots within each allowed region indicate the position of the local best-fit point in parameter space. The results shown in Fig. 2 were calculated using the predictions of 
the 1998 standard solar model of Bahcall and Pinsonneault [20], which includes helium and heavy element diffusion and uses the recent reevaluation of solar fusion cross sections [21]; the shape of the allowed contours depends only slightly upon the assumed solar model (see Fig. 1 of Ref. [31]).

The BOREXINO experiment will measure the $\nu-e$ scattering rate for the $0.86 \mathrm{MeV}{ }^{7} \mathrm{Be}$ line. We have calculated the allowed range for the scattering rate at $99 \%$ C.L. for the different MSW solutions discussed above. The rates are given in the following equation relative to the 1998 Bahcall and Pinsonneault standard model [20] are:

$$
\begin{gathered}
\frac{\langle\phi \sigma\rangle_{{ }^{7} \mathrm{Be} \mathrm{SMA}}}{\langle\phi \sigma\rangle_{\mathrm{BP} 98}}=0.23_{-0.01}^{+0.24} \\
\frac{\langle\phi \sigma\rangle^{7} \mathrm{Be} \mathrm{LMA}}{\langle\phi \sigma\rangle_{\mathrm{BP} 98}}=0.59_{-0.18}^{+0.15}, \\
\frac{\langle\phi \sigma\rangle_{{ } \mathrm{Be} \mathrm{LOW}}}{\langle\phi \sigma\rangle_{\mathrm{BP} 98}}=0.59_{-0.08}^{+0.06} .
\end{gathered}
$$

\section{Nuclear physics uncertainties}

After more than 35 years of progressively more accurate measurements of input parameters and more precise solar modeling, the largest recognized uncertainties that afflict the prediction of solar neutrino fluxes are associated with the laboratory cross sections at low energies for the crucial ${ }^{3} \mathrm{He}(\alpha, \gamma){ }^{7} \mathrm{Be}$ and the ${ }^{7} \mathrm{Be}(p, \gamma){ }^{8} \mathrm{~B}$ reactions [20]. The uncertainty in the ${ }^{3} \mathrm{He}-{ }^{4} \mathrm{He}$ cross section is $9.4 \%, 1 \sigma$ [21]. The uncertainty in the ${ }^{7} \mathrm{Be}+p$ reaction is asymmetric and is, on average, $10.6 \%$ [21]. The flux of ${ }^{8} \mathrm{~B}$ neutrinos is directly proportional to the rate of the ${ }^{7} \mathrm{Be}+p$ reaction and the flux of the ${ }^{7} \mathrm{Be}$ neutrinos is approximately linearly proportional to the rate of the ${ }^{3} \mathrm{He}-{ }^{4} \mathrm{He}$ reaction.

How much do the uncertainties in the nuclear physics parameters affect the accuracy with which one can determine neutrino parameters? In order to answer this question, we have calculated the allowed regions for MSW solutions, analogous to those considered in Sec. IVA1 , but we assumed in the present case either that the uncertainty in the ${ }^{3} \mathrm{He}(\alpha, \gamma){ }^{7} \mathrm{Be}$ or the ${ }^{7} \mathrm{Be}(p, \gamma){ }^{8} \mathrm{~B}$ reaction was equal to zero.

Figure 3 shows that the MSW allowed regions are reduced only slightly when the uncertainties associated with either the ${ }^{3} \mathrm{He}(\alpha, \gamma){ }^{7} \mathrm{Be}$ (cross-section factor $S_{34}$ ) or the ${ }^{7} \mathrm{Be}(p, \gamma)^{8} \mathrm{~B}$ reaction (cross-section factor $S_{17}$ ) are artificially decreased to zero. Comparing Fig. 3 and Fig. 2, we see that in both cases the allowed regions for MSW solutions remain comparable in size to what they are calculated to be with realistic estimates of the nuclear physics uncertainties. The reason that the allowed regions are not particularly sensitive to the uncertainties in any one nuclear parameter is that there are a number of roughly comparable uncertainties from different input parameters [20].

We conclude that one cannot greatly increase the accuracy with which MSW parameters can be determined by greatly decreasing the uncertainty in any single input parameter. 


\section{Sterile neutrinos}

How are the results given above changed if the oscillations involve sterile neutrinos?

Figure 4 shows that if the oscillations are between an electron-type neutrino and a sterile neutrino then the LMA and the LOW solutions are not allowed at the 99\% C.L. ; only the SMA solution is possible. The LMA and LOW solutions are ruled out at a very high C.L.; the value of $\chi^{2}(\mathrm{~min})=19.0$ (17.0) for the LMA (LOW) solutions. However, the SMA solution is still allowed, with $\chi^{2}(\mathrm{~min})=1.7$ (which is the same as for the best-case with active neutrinos) and the best-fit solution is

$$
\begin{gathered}
\Delta m^{2}=4.3 \times 10^{-6} \mathrm{eV}^{2}, \\
\sin ^{2} 2 \theta=6.9 \times 10^{-3} .
\end{gathered}
$$

The mass difference for the solution involving sterile neutrinos, Eq. (8a), is slightly smaller than the mass difference found for oscillations between active neutrinos (cf. Eq. 19a), but the mixing angle for sterile neutrinos, Eq. Bb, is slightly larger than for active neutrinos (cf. Eq. (4b).

The suppression of the neutrino fluxes in the case of SMA conversion into sterile neutrinos is somewhat similar to the arbitrary reductions of the neutrino fluxes considered in Sec. IIB. The flux of $p p$ neutrinos is unsuppressed, the ${ }^{7} \mathrm{Be}$ neutrino flux as well as other fluxes of intermediate energies are strongly suppressed, and the ${ }^{8} \mathrm{~B}$ neutrino flux is moderately suppressed. The fit to the solar neutrino experiments using oscillations into sterile neutrinos is much better than the fit to the experiments made using an arbitrary linear combination of undistorted solar neutrino fluxes (cf. Sec. IIB). The main difference between the suppression due to sterile neutrinos and the suppressions considered in Sec. IIB is that oscillations into sterile neutrinos suppress the lower energy part of the ${ }^{8} \mathrm{~B}$ neutrino energy spectrum. This result is an illustration of our claim made in Sec. IIO that the evidence from the total experimental rates suggests the existence of a distortion of the solar neutrino energy spectrum.

What should one expect for BOREXINO if oscillations involve sterile neutrinos? The allowed range for the ${ }^{7} \mathrm{Be}$ electron scattering rate is

$$
\frac{\langle\phi \sigma\rangle_{7_{\text {Be sterile }}}}{\langle\phi \sigma\rangle_{\mathrm{BP} 98}}=0.009_{-0.005}^{+0.244} .
$$

If oscillations occur to sterile neutrinos, the rate observed in BOREXINO will be - for almost the entire range of allowed parameters - less than the lowest expected rate if oscillations occur to active neutrinos (cf. Eq. \&).

If the Caltech normalization for the ${ }^{8} \mathrm{~B}$ production cross section $\left(S_{17}=22.4 \mathrm{kev} \mathrm{b}\right)$ is used instead of the INT normalization, the allowed regions are shifted only slightly from what they are in Figure 2 and Figure 1 . The best estimate solutions for $\delta m^{2}$ are changed by less than $7 \%$ relative to the values given in Eqs. (44)-(6) and Eq. (8). 


\section{B. Vacuum neutrino oscillations}

Figure 5 shows for vacuum neutrino oscillations the broad region of solutions allowed at 99\% C.L. . The best-fit vacuum solution is

$$
\begin{gathered}
\Delta m^{2}=8.0 \times 10^{-11} \mathrm{eV}^{2}, \\
\sin ^{2} 2 \theta=0.75,
\end{gathered}
$$

which has a $\chi_{\min }^{2}=4.3$.

What will BOREXINO observe if vacuum neutrino oscillations occur? The allowed range for vacuum oscillations of the ${ }^{7} \mathrm{Be}$ line is very large if the only constraints imposed are consistent with the total observed rates. We find at $99 \%$ C.L. that

$$
\frac{\langle\phi \sigma\rangle_{7 \mathrm{Be}} \text { vacuum }}{\langle\phi \sigma\rangle_{\mathrm{BP} 98}}=0.46_{-0.18}^{+0.46} \text {. }
$$

There is no allowed solution at the $99.7 \%$ C.L. for vacuum neutrino oscillations between $\nu_{e}$ and a sterile neutrino. The solution involving sterile neutrinos has $\chi^{2}(\min )=12.0$.

\section{Arbitrary ${ }^{8} \mathrm{~B}$ neutrino flux}

The value of the ${ }^{8} \mathrm{~B}$ neutrino flux calculated in the standard solar model (cf. Ref. [20]) is more uncertain $(+19 \%$ and $-14 \%, 1 \sigma)$ than any of the other experimentally-important solar

neutrino fluxes. It is therefore useful to consider what constraints are placed upon neutrino physics if the ${ }^{8} \mathrm{~B}$ flux is treated as a free parameter 48,33 .

\section{MSW solutions}

Figure 6 shows the allowed parameter space for MSW oscillations when the ${ }^{8} \mathrm{~B}$ flux is allowed to take on arbitrary values. The best-fit SMA solution is

$$
\begin{aligned}
\Delta m^{2} & =5.0 \times 10^{-6} \mathrm{eV}^{2}, \\
\sin ^{2} 2 \theta & =3.5 \times 10^{-3},
\end{aligned}
$$

which has $\chi_{\min }^{2}=0.86$. The best-fit for the LMA solution is

$$
\begin{aligned}
\Delta m^{2} & =1.6 \times 10^{-5} \mathrm{eV}^{2}, \\
\sin ^{2} 2 \theta & =0.57,
\end{aligned}
$$

which has $\chi_{\min }^{2}=0.91$. The LOW solution,

$$
\begin{aligned}
\Delta m^{2} & =7.9 \times 10^{-8} \mathrm{eV}^{2}, \\
\sin ^{2} 2 \theta & =0.95,
\end{aligned}
$$


has a much larger $\chi_{\min }^{2}=7.2$. For all three classes of MSW solutions the squared mass differences are changed by less than $20 \%$ if the ${ }^{8} \mathrm{~B}$ neutrino flux is treated as a free parameter (cf. Eq. 1 - Eq. 6), although the values of $\sin ^{2} 2 \theta$ are changed by much large factors (a factor of two for the SMA solution).

Do the solar neutrino observations place useful limits on the value of the cross section factor, $S_{17}$, for the production of ${ }^{8} \mathrm{~B}$ ?

Figure 7 shows the minimum value of $\chi^{2}$ obtained using as constraints the rates of the solar neutrino experiments but allowing the value of the ${ }^{8} \mathrm{~B}$ flux to take on arbitrary values. The allowed range at $99 \%$ C.L. is: $0.4<S_{17} / S(\mathrm{INT})_{17}<2.0$, which is much broader than the range allowed [21] by direct laboratory experiments.

If oscillations between sterile neutrinos are considered, the constraints set on $S_{17}$ by varying the solar ${ }^{8} \mathrm{~B}$ flux and fitting to the solar neutrino data are again much larger than the uncertainty in the laboratory measurement of $S_{17}$ (cf. Ref 44]).

\section{Vacuum oscillations}

How are the inferred parameters for vacuum neutrino oscillations affected by permitting arbitrary values for the assumed ${ }^{8} \mathrm{~B}$ neutrino flux, cf. Ref. [49]?

Figure 8 shows the expanded solution space that is allowed for vacuum oscillations if the ${ }^{8} \mathrm{~B}$ neutrino flux is unconstrained. Instead of the rather limited parameter space that is permitted with the standard solar model value of the ${ }^{8} \mathrm{~B}$ flux (see Fig 5 ), the squared neutrino mass difference can span the entire two order of magnitude range from $\Delta m^{2}=4 \times 10^{-12} \mathrm{eV}^{2}$ to $\Delta m^{2}=5 \times 10^{-10} \mathrm{eV}^{2}$ if $S_{17}$ is allowed to vary arbitrarily. The allowed range of the ${ }^{8} \mathrm{~B}$ cross section factor at $99 \%$ C.L. is, for vacuum oscillations, $0.4<S_{17} / S(\text { INT })_{17}<2.0$.

For completeness, we note that the best-fit values for the vacuum solution and arbitrary ${ }^{8} \mathrm{~B}$ flux are

$$
\begin{gathered}
\Delta m^{2}=8.4 \times 10^{-11} \mathrm{eV}^{2}, \\
\sin ^{2} 2 \theta=0.98,
\end{gathered}
$$

which has a shallow $\chi_{\min }^{2}=0.94$. The minimum occurs for a ${ }^{8} \mathrm{~B}$ flux that is 1.9 times the value of the predicted BP98 ${ }^{8} \mathrm{~B}$ flux.

This allowed range of $S_{17}$ is very similar for both the MSW and the vacuum solutions. In both cases, the measurements by SuperKamiokande determine the upper and lower limits on the allowed ${ }^{8} \mathrm{~B}$ flux. The lower limit corresponds to very little conversion from $\nu_{e}$ to other neutrino types. The lowest allowed value is obtained by reducing the best-estimate SuperKamiokande flux by the $3 \sigma$ experimental uncertainty. Since the experimental uncertainty is small $\left(1 \sigma_{\exp }=3 \%\right)$, the lower limit is close to the SuperKamiokande best-estimate value. The upper limit is much larger because essentially all of the ${ }^{8} \mathrm{~B}$ flux observed by SuperKamiokande is $\nu_{\mu}$ or $\nu_{\tau}$. Since the cross section [18 for electron-neutrino scattering by $\nu_{\mu}$ or $\nu_{\tau}$ is about six times smaller than the scattering cross section for $\nu_{e}$, and $\nu_{\mu}$ and $\nu_{\tau}$ are not detected in the chlorine and gallium experiments, the upper limit for the total flux can be much larger (a factor of five in practice) than the observed flux. 


\section{Energy-independent suppression of neutrino fluxes}

Many authors have considered particle-physics models which predict energy-independent reductions of the solar neutrino fluxes (see, e. g., Ref. [50 54] and references cited therein). In these scenarios, all neutrino fluxes are reduced by some particle-physics mechanism by exactly the same factor. One can test the goodness-of-fit of such scenarios by calculating the minimum $\chi^{2}$ for this case [55]. Using the predictions and the uncertainties in the BP98 solar model and the observed rates and their uncertainties given in Table $\mathbb{I}$, we have calculated the minimum $\chi^{2}$ for different neutrino oscillation scenarios.

For arbitrary reduction factors $\alpha$, the best-fit value is $\alpha=0.48$ for oscillation into active neutrinos and $\alpha=0.50$ for sterile neutrinos. The minimum $\chi_{\min }^{2}=12.0$ for active neutrinos and $\chi_{\min }^{2}=19.3$ for sterile neutrinos. Since there are two degrees of freedom in these cases, energy-independent oscillation into active neutrinos is ruled out at the 99.8\% C.L. and energy-independent oscillation into sterile neutrinos is ruled out at the 99.99\%C.L. . These results are changed only slightly (strengthened slightly) if the ${ }^{8} \mathrm{~B}$ flux is allowed to vary independently of all other fluxes. In this case, we obtain for active neutrinos (sterile neutrinos $) \chi_{\min }^{2}=11.0\left(\chi_{\min }^{2}=19.3\right)$ for 1 d.o.f., which is excluded at the $99.8 \%$ C.L. (99.999\% C.L.) .

For the model considered in Ref. [51], $\alpha=5 / 9$. In this case, $\chi_{\min }^{2}=14.3$ (3 d.o.f.), and the model is ruled out at the $99.8 \%$ C. L. .

\section{E. Summary of analysis of rates}

The principal results of this section are displayed in Fig. 2-Fig. 8, except for the BOREXINO predictions which are given in Eq. (7), Eq. (9), and Eq. (11), and the demonstration in Sec. IVD that constant-reduction solutions are disfavored. For the reader's convenience, we also present the numerical values of the best-fit neutrino mixing angles and mass differences for each case considered.

In all of the calculations shown in the figures, we have used the INT normalization for the ${ }^{8} \mathrm{~B}$ production cross section, $S_{17}$. However, this cross section is relatively poorly known and, unfortunately, at present it is largely a matter of judgment as to which normalization, INT or CIT, is most appropriate. We have therefore presented results for both normalizations; the difference between neutrino parameters determined with the INT and the CIT normalizations is an indication of the magnitude of the uncertainties in neutrino parameters that are caused by uncertainties in the basic nuclear physics data.

Particle physics solutions in which the suppression of the electron neutrino flux is independent of the neutrino energy are ruled out at more than the $99 \% \mathrm{C}$. L. for oscillations into both active and sterile neutrinos (see Sec. IVD).

Table II presents the minimum $\chi^{2}$ for the different neutrino oscillation scenarios that we have considered in this section. The primary entries in the table represent $\chi^{2}(\mathrm{~min})$ with the INT normalization for $S_{17}$; the entries enclosed in parentheses were computed using the CIT normalization for $S_{17}$. The INT and CIT normalizations give about equally good fits to the observed rates in solar neutrino experiments, with the INT normalization slightly favoring the SMA MSW solution and the CIT normalization slightly favoring the LMA solution (see also Fig. 7). Indeed, the larger ${ }^{8} \mathrm{~B}$ flux, the CIT normalization, implies a stronger suppression 
by oscillations at higher energies, and consequently, a flatter dependence of the suppression factor on energy than is implied by the INT normalization.

\section{ZENITH-ANGLE DEPENDENCE OF RATES}

If MSW oscillations occur, the observed event rates in solar neutrino experiments can depend upon which region, if any, of the earth that the neutrinos traverse before reaching the detector. At times when the sun is below the horizon, $\nu_{\mu}$ or $\nu_{\tau}$ coming from the sun can be re-converted in the earth into the more easily detected $\nu_{e}$. Thus for a certain range of neutrino parameters the observed event rate will depend upon the zenith angle of the sun, i.e., the angle that the sun makes with respect to the direction of the zenith at the position of the neutrino detector. This is known as the earth regeneration effect [56 58].

\section{A. Expectations for survival probabilities}

Figure 9 shows the computed survival probabilities for electron type neutrinos as a function of energy for the day (no regeneration), the night (with regeneration), and the annual average. The survival probabilities computed here for the best-fit points differ relatively little from the values calculated earlier (see Fig. 4 of Ref. [43]), despite the fact that in the present study we have taken account of the measured SuperKamiokande rate, the somewhat different fluxes from the BP98 model, the improved estimates for nuclear fusion cross sections, and other updated data. The changes in the best-fit oscillation parameters caused by the use of the new data are relatively small (see Sec. [V). The principal difference is that with the present parameters the expected Day-Night difference for the SMA solution is extremely small, not even visible in Fig. 9. Note, however, that a potentially detectable Day-Night difference is predicted for the LMA solution at the higher neutrino energies. The Day-Night difference is expected to be relatively large for the LOW solution, but only at energies below $1 \mathrm{MeV}$. The Day-Night difference predicted by the LOW solution would be detectable by the BOREXINO experiment, which will observe the $0.86 \mathrm{MeV}$ neutrino line from ${ }^{7}$ Be electron capture.

\section{B. SuperKamiokande result}

The SuperKamiokande Collaboration has given a preliminary value for the difference between the event rates at night and during the day. After only 504 days of data, they obtain an initial estimate for this Day-Night asymmetry, $A$, of [8, 10]:

$$
A=\frac{D-N}{D+N}=-0.023 \pm 0.020 \text { (stat) } \pm 0.014 \text { (syst) }
$$

This estimate applies for events in which the recoil electron has an energy of at least 6.5 $\mathrm{MeV}$. The difference shown in Eq. (16) is in the direction that would be expected from regeneration in the earth (the sun is apparently brighter at night in neutrinos), but is small and is not statistically significant. 
The SuperKamiokande Collaboration has also given [8,10] a preliminary distribution of the event rates versus zenith angle in which the rates and their errors are plotted in 10 angular bins.

\section{Constraints on neutrino parameters}

We have determined the constraints placed upon neutrino mixing parameters using the preliminary Day-Night asymmetry (Eq. 16) and also the 10-bin zenith angle distribution. We use the techniques and results contained in our previous detailed discussion of the expected zenith-angle dependence of solar event rates [43] to analyze the 10 bin measurements of the zenith angle distribution reported by the SuperKamiokande Collaboration for the first 504 days of observations [10].

Figure 10a shows the results of a combined MSW $\chi^{2}$ fit with active neutrinos of the SuperKamiokande zenith angle dependence and the total rates of the Homestake, GALLEX, SAGE, and SuperKamiokande experiments. The region in the figure that is excluded by the zenith angle dependence is shaded lightly and, almost touches the darkly shaded LMA and SMA allowed regions for the combined zenith angle and rate constraints. Figure 10b shows the combined solutions using the SuperKamiokande Day-Night asymmetry and the four total rates. As shown previously from simulated data [43], the Day-Night asymmetry is more sensitive to the LMA solution and the zenith-angle distribution is more sensitive to the SMA solution. We see from Fig. 10b that the observations by SuperKamiokande of the Day-Night asymmetry have eliminated a sizable fraction, almost a half, of the parameter space for the LMA solution that is allowed if one only considers the total rates (cf. Fig. 2). A small part (with $\sin ^{2} 2 \theta>10^{-2}$ ) of the parameter space of the SMA solutions is excluded by the measured zenith-angle dependence.

Figure 11a and Figure 11]b show the analogous regions for MSW oscillations into sterile neutrinos. The only MSW solution for oscillation into sterile neutrinos is the SMA. The regeneration effect is not as effective a test of the sterile neutrino oscillation solution as it is for oscillation into active neutrinos (cf. Fig. 11 and Fig. 2).

\section{PRELIMINARY SPECTRAL SHAPE}

If minimal standard electroweak theory is correct, the shape of the ${ }^{8} \mathrm{~B}$ neutrino energy spectrum is independent of all solar influences to an accuracy of 1 part in $10^{5}$ [59]. The shape of the neutrino spectrum determines the shape of the recoil electron energy spectrum produced by neutrino-electron scattering in the detector. Therefore, any departure of the observed electron recoil energy spectrum from the shape predicted by standard electroweak theory would be a "smoking gun" indication of new physics. In this section, we compare the preliminary recoil electron energy spectrum reported by SuperKamiokande [10] with the results calculated using the standard (undistorted) ${ }^{8} \mathrm{~B}$ [19] neutrino spectrum. We also compare the observed energy spectrum with recoil energy spectra calculated assuming MSW or vacuum neutrino oscillations and use these results to constrain the allowed neutrino parameter space. 


\section{A. SuperKamiokande energy spectrum}

The SuperKamiokande Collaboration has made available 10 preliminary data, including estimated statistical and systematic uncertainties, from 504 days of operation in which the recoil energy spectrum is divided into 16 bins, with 15 bins having a width of 0.5 $\mathrm{MeV}$ starting at $6.5 \mathrm{MeV}$ and continuing to $14 \mathrm{MeV}$. The final bin includes events with energies from $14 \mathrm{MeV}$ to $20 \mathrm{MeV}$ and contains more counts than would be expected from an undistorted ${ }^{8} \mathrm{~B}$ spectrum normalized at lower energies.

\section{B. Fit to undistorted energy spectrum}

The minimum $\chi^{2}$ for the fit of the undistorted energy spectrum to the measured energy spectrum is 31 for 15 D.O.F. . The fit with the undistorted spectrum is acceptable only at slightly less than the 1\% C.L., consistent with the results reported by the SuperKamiokande Collaboration at Neutrino 98 [10].

\section{Fits to MSW and vacuum oscillations}

The simplest test [60] for a deviation from the standard recoil energy spectrum is to investigate whether the ratio, $R$, of the observed to the standard energy spectrum is a constant, which would be expected in the absence of a distortion. We have therefore fit the ratio $\mathrm{R}$ to a linear function using the measured [10 number of events and their quoted uncertainties in the 16 bins (from $6.5 \mathrm{MeV}$ to $20 \mathrm{MeV}$ ) and the standard electron recoil energy spectrum [19] modified by the energy resolution of the SuperKamiokande detector. Thus

$$
R=R_{0}+S_{0} *\left(W_{e}-10 \mathrm{MeV}\right)
$$

where $R_{0}$ represents the average event rate and $S_{0}$ is the average slope that measures the deviation of the recoil electron energy spectrum from the undistorted shape. The total electron energy is $W_{e}$. The energy spectrum of recoil electrons is determined by convolving the neutrino spectrum [19] with the calculated survival probability, the neutrino-electron scattering cross-sections [18], and the energy resolution function [10]. These integrations smooth the effect of distortions; therefore, the expected distortion can be well described by a simple linear dependence.

Figure 12 shows the result of this calculation. The $1 \sigma, 2 \sigma$, and $3 \sigma$ allowed regions are shown in the figure. We have taken account of the bin correlations between the systematic errors in the fashion explained in the Appendix. The best-fit point lies at $R_{0}=0.474$ and $S_{0}=0.0153$, with $\chi_{\min }^{2}=23.5$ for 14 d.o.f.. Therefore, a straight line is not a particularly good fit to the data; it is acceptable at the $5.3 \%$ C.L. . The main reason that the fit is acceptable only at a modest C.L. is that the last three bins indicate a deviation from a smooth extrapolation at lower energies. This deviation could be real or it could be a statistical fluctuation.

Figure 12 also shows the ranges of the predicted slopes $\left(S_{0}\right)$ for different successful descriptions of the solar neutrino total experimental rates, i.e., the SMA active and sterile, 
LMA, LOW, and vacuum oscillation solutions described in Sec. V. All of the oscillation solutions shown in Fig. 12 permit a wide range of $R_{0}$ that includes the range measured by the SuperKamiokande Collaboration. For visual convenience, we have not included the horizontal error bars, i. e., the uncertainties in $R_{0}$, for the neutrino oscillation solutions shown in Fig. 12. Therefore, there are acceptable oscillation solutions of all four types that describe both the total rates in the four solar neutrino experiments and the recoil electron energy spectrum measured by SuperKamiokande.

How powerful a constraint is the spectrum shape in determining the allowed neutrino oscillation parameters?

Figure 13 shows, for both active and sterile neutrinos, the parameter region that is allowed by considering only the spectral information. Only a small fraction of MSW parameter space is consistent with the spectral data. It is instructive to compare the regions allowed by the rates only, shown in Fig. 2 and Fig. 目, with the regions shown in Fig. 13, which are allowed by the spectrum data. The best-fit solutions considering only the spectrum, marked by dark points in Fig. 13, do not lie within the allowed regions for the global solutions discussed in Sec. VIT.

Figure 14 shows the region that is allowed for vacuum oscillations by the information from the SuperKamiokande energy spectrum. The dark circle shows the best-fit point. Just as for MSW oscillations, only a small fraction of the parameter space is allowed for vacuum oscillations by the spectrum constraint. The complementarity of the analysis that uses only

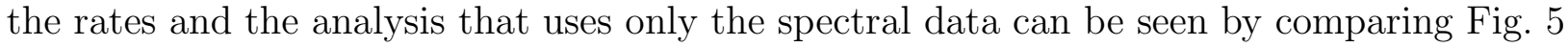
and Fig. 14 .

\section{GLOBAL SOLUTION: RATES, ZENITH-ANGLE DISTRIBUTION, AND ENERGY SPECTRUM}

We discuss in this section simultaneous fits to all the available data. We consider solutions that describe the total rates in the four experiments (chlorine, SuperKamiokande, GALLEX, and SAGE) plus either the energy spectrum measured by SuperKamiokande or both the energy spectrum and the Day-Night asymmetry (or zenith-angle dependence) measured by SuperKamiokande.

We begin by showing in Sec. VIIA that an arbitrary combination of undistorted solar neutrino energy spectra is, independent of any astrophysical considerations, ruled out at the $3.5 \sigma$ level. We next show in Sec. VIIB that the best global fit for the MSW solutions is acceptable at the $7 \%$ C.L. for active neutrinos (8\% C.L. for sterile neutrinos). The best-fit global solution is very close the best-fit SMA solution when only the total experimental rates are considered. Finally, we show in Sec. VIIC that the best global fit for vacuum oscillations is acceptable at the $5 \%$ C.L. .

\footnotetext{
$\ddagger$ The allowed range, cf. Sec. IV], of $R_{0}$ is 0.38 to 0.86 for the SMA active solution, 0.33 to 0.84 for the SMA sterile neutrin solution, 0.31 to 0.55 for the LMA solution, and 0.42 to 0.53 for the LOW solution.
} 
The results of the simultaneous fits to all of the available data of the neutrino predictions are shown in Fig. 15-Fig. 17, which present the allowed regions for the different oscillation scenarios.

\section{A. Global fits: arbitrary undistorted fluxes}

What is the best fit to the total rates plus the SuperKamiokande spectrum and DayNight asymmetry if we allow arbitrary values, subject only to the luminosity constraint [31, for the $p p,{ }^{7} \mathrm{Be},{ }^{8} \mathrm{~B}$, and CNO fluxes ? The minimum $\chi^{2}$ satisfies

$$
\chi_{\text {minimum }}^{2}\left(3 \text { rates }+ \text { spectrum }+\mathrm{D} / \mathrm{N} ; \text { arbitrary } p p,{ }^{7} \mathrm{Be},{ }^{8} \mathrm{~B},{ }^{13} \mathrm{~N},{ }^{15} \mathrm{O}\right)=39.2,
$$

for 15 d.o.f.] This result is excluded at the $99.94 \%$ C.L., which corresponds to a $3.5 \sigma$ deviation from minimal electroweak theory.

\section{B. Global fits: MSW solutions}

Fig. 15a displays the results of imposing on the MSW solutions the combined constraints of the total rates and the SuperKamiokande spectrum. The best-fit MSW solution considering both total rates in the four experiments and the electron recoil energy spectrum measured by SuperKamiokande has

$$
\begin{gathered}
\Delta m^{2}=5.4 \times 10^{-6} \mathrm{eV}^{2}, \\
\sin ^{2} 2 \theta=6.3 \times 10^{-3} .
\end{gathered}
$$

The minimum $\chi_{\min }^{2}=26.5$, which is acceptable at the $7 \%$ C.L. (for 17 d.o.f.), not a very good fit. The best-fit solution is very close to the SMA solution when only rates are considered (cf. Eq. (4). Including the spectrum in addition to the total rates, eliminates (at 99\% C.L.) the entire LOW solution and a large portion of the LMA solution at higher $\Delta m^{2}$. The inclusion of the spectral constraint also eliminates for the SMA solution part of the region at smaller mixing angles that is allowed if only the total rates are considered.

Fig. 15b shows the allowed region for MSW parameters when the constraints from the SuperKamiokande zenith-angle distribution is included together with the constraints from the four measured total rates and the SuperKamiokande electron recoil energy spectrum.

$\S$ There are 3 d.o.f associated with the rates, 15 d.o.f associated with the spectra shape which has one overall normalization parameter that is variable, and 1 d.o.f. for the Day-Night asymmetry. All 5 fluxes are allowed to vary freely, subject only to the luminosity constraint.

**We have not shown the combined fit including the Day-Night asymmetry, the spectrum constraint, and the total experimental rates since the excluded region is very similar to what appears in Fig. 15b. We chose to display the fit with the zenith-angle distribution since this provides the best restriction on the SMA solution. 
The best-fit solution is almost identical to what is obtained for the rates only case and for the case of rates plus zenith-angle constraint, namely,

$$
\begin{gathered}
\Delta m^{2}=5.4 \times 10^{-6} \mathrm{eV}^{2}, \\
\sin ^{2} 2 \theta=5.5 \times 10^{-3} .
\end{gathered}
$$

The minimum $\chi_{\min }^{2}=37.2$, which again is acceptable at the $7 \%$ C.L. (for 26 d.o.f.). Only the SMA solution survives at the $99 \%$ C.L. when the zenith-angle and the spectrum constraints are added to the constraints of the total experimental rates. The LMA and the LOW solution are marginally ruled out $\left(\chi_{\min }^{2}=47(49)\right.$ for the LMA (LOW) solution).

The Day-Night asymmetry parameter, $A$, which is defined by Eq. (16), lies in the range

$$
-0.0048<A<0.025,
$$

for active neutrinos when all three sets of constraints are applied. The best-fit solution predicts a Day-Night asymmetry whose absolute value is less than $0.1 \%$.

How is the global solution changed if oscillations involve sterile neutrinos? Figure 16 shows the allowed solution space when the measured total rates, energy spectrum, and zenith-angle dependence are all included as constraints. The best-fit solution is very close to what is obtained from the rates only (or the rates plus spectrum constraints) for sterile neutrinos. We find

$$
\begin{gathered}
\Delta m^{2}=4.0 \times 10^{-6} \mathrm{eV}^{2}, \\
\sin ^{2} 2 \theta=6.9 \times 10^{-3} .
\end{gathered}
$$

The minimum $\chi_{\min }^{2}=36.5$, which is acceptable at the $8 \%$ C.L. (for 26 d.o.f.), slightly better than for active neutrinos. For sterile neutrinos, the Day-Night asymmetry parameter, $A$, lies in the range

$$
-0.0031<A_{\text {sterile }}<0.007 \text {. }
$$

The best-fit solution predicts a value for the asymmetry parameter whose absolute value is less than $0.1 \%$.

\section{Global fits: vacuum oscillations}

Fig. 17 shows the allowed regions for the vacuum oscillation parameters when the constraints from the rates, the spectrum shape, and the Day-Night asymmetry are all included. The best-fit vacuum oscillation solution has

$$
\begin{gathered}
\Delta m^{2}=6.5 \times 10^{-11} \mathrm{eV}^{2}, \\
\sin ^{2} 2 \theta=0.75 .
\end{gathered}
$$

The minimum $\chi_{\min }^{2}=28.4$, which is acceptable at the $6 \%$ C.L. (for 18 d.o.f.). This value of $\chi_{\min }^{2}$ is only slightly below the value of $\chi^{2}=30.5$ that is found for the best-fit point in the 'spectrum-only' analysis (cf. Fig. 14). The inclusion of the spectral constraints reduces considerably the domain of allowed solutions for vacuum oscillations (cf. Fig. 17 and Fig. 5). 


\section{Global fits: predicted ${ }^{7} \mathrm{Be} \nu-e$ scattering rates}

We have calculated the range of allowed ${ }^{7} \mathrm{Be}$ neutrino-electron scattering rates $(0.86 \mathrm{MeV}$ line) that is consistent with all of the available solar neutrino data: total event rates, DayNight asymmetry, and spectrum shape. The results are very similar to what was obtained earlier in the discussion of the rates only, see Eq. (7), Eq. (9), and Eq. (11). We conclude that the neutrino oscillation predictions for the BOREXINO experiment are robust. We find, in particular, that the global range predicted by the MSW solution with active neutrinos is

$$
\frac{\langle\phi \sigma\rangle_{{ }^{7} \mathrm{Be} \text { SMA }}}{\langle\phi \sigma\rangle_{\mathrm{BP} 98}}=0.23_{-0.01}^{+0.24} \text {. }
$$

The corresponding range for MSW oscillations into sterile neutrinos is

$$
\frac{\langle\phi \sigma\rangle_{{ }^{7} \mathrm{Be} \text { sterile }}}{\langle\phi \sigma\rangle_{\mathrm{BP} 98}}=0.006_{-0.002}^{+0.25} \text {. }
$$

For vacuum oscillations,

$$
\frac{\langle\phi \sigma\rangle_{{ } \mathrm{Be} \text { vacuum }}}{\langle\phi \sigma\rangle_{\mathrm{BP} 98}}=0.45_{-0.11}^{+0.33}
$$

The principal change caused by the imposition of the additional constraints due to the measured spectrum shape and Day-Night asymmetry is a modest shrinking of the allowed range for the vacuum solutions.

\section{E. Global fits: predicted ${ }^{7} \mathrm{Be} \nu_{e}$ fluxes}

Raghavan 61] has recently proposed a flavor-specific (neutrino-absorption) experiment that measures the total flux of $0.86 \mathrm{MeV} \nu_{e}$ reaching the earth from ${ }^{7}$ Be electron captures in the sun. The preliminary name for this experiment is LENS.

We have calculated the range of allowed ${ }^{7} \mathrm{~B} \nu_{e}$ survival probabilities for comparison with the results of a future LENS experiment. We again considered neutrino parameters consistent with all the available solar neutrino data: total event rates, Day-Night asymmetry, and spectrum shape. We give below the predicted values for the survival probability, $P$, for an electron-type $0.86 \mathrm{MeV}$ neutrino created in sun to remain an electron-type neutrino when it reaches the terrestrial target. For MSW oscillations into active neutrinos we find

$$
P_{7_{\mathrm{Be}} \mathrm{SMA}}=0.02_{-0.01}^{+0.30} \text {. }
$$

The corresponding range for MSW oscillations into sterile neutrinos is

$$
P_{7 \text { Be sterile }}=0.006_{-0.002}^{+0.25} \text {. }
$$

For vacuum oscillations,

$$
P_{7 \text { Be vacuum }}=0.30_{-0.14}^{+0.42} \text {. }
$$

With current knowledge, the allowed range of $P$ varies all the way from 0.00 to 0.72 , which emphasizes the importance of this proposed measurement. 


\section{F. Global fits: energy-independent suppression}

The preliminary results of the SuperKamiokande measurement of the electron recoil energy spectrum provide additional evidence, beyond that available from just the total rates discussed in Section IVD, regarding the hypothesis [50 54] for an energy-independent suppression of the solar neutrino fluxes (but see also the discussion of Figure 18 in Section VIIIE) Assuming an energy-independent suppression of the neutrino fluxes in the BP98 standard solar model [20], $\chi_{\min }^{2}$ for oscillations into active neutrinos is 43 (17 d.o.f), i.e., this solution is disfavored at $99.95 \%$ C.L. . If the ${ }^{8} \mathrm{~B}$ neutrino flux is allowed to vary as a free parameter, $\chi_{\min }^{2}=42$ ( 16 d.o.f), which is disfavored at $99.96 \%$ C.L. . Energy-independent oscillations into sterile neutrinos are disfavored at 99.998\% C.L. (minimum $\chi^{2}=50.3$ ) if one allows an arbitrary ${ }^{8} \mathrm{~B}$ neutrino flux. Maximally-mixed oscillations into active neutrinos which predict [51 a constant survival probability of 5/9 give $\chi_{\min }^{2}=45$ (18 d.o.f.), which is disfavored at $99.96 \%$ C.L. .For arbitrary ${ }^{8} \mathrm{~B}$ neutrino flux, $\chi^{2}=44.5$ (17 d.o.f), which is disfavored at $99.97 \%$ C.L. .

\section{DISCUSSIONS AND CONCLUSIONS}

We summarize and discuss in this section our principal conclusions. In Sec. VIIIA, we discuss the indications that solar neutrino experiments are suggesting new physics. We present in Sec. VIIIB the robust inferences based only on the total event rates and in Sec. VIIIC the implications of the absence of a statistically significant zenith-angle dependence for the SuperKamiokande event rates. In Sec. VIIID, we analyze the strong constraints imposed by the electron recoil energy spectrum measured by SuperKamiokande. Our global analysis is described in Sec. VIIIE, where we present the implications of the combined constraints from the total rates, the zenith-angle dependence, and the spectrum shape. We summarize in Sec. VIIIF our overall view of the solar neutrino situation.

For a given hypothesis, MSW or vacuum oscillations (active or sterile neutrinos), we search numerically for the best-fit solution and quote the C.L. for acceptance or exclusion based upon the relevant $\chi_{\min }^{2}$ and the appropriate number of d.o.f. . The acceptance levels found in this paper seem somewhat higher than reported by the SuperKamiokande Collaboration in their review talk at Neutrino 98, but we think that this difference is largely due to the fact that we have searched for the best-fit solution rather than test the acceptability of previously recognized solutions and that we have used the BP98 rather than the BP95 predictions in analyzing the total rates. To the best of our knowledge, the present paper is the first to determine global solutions that take account of the measured total rates in all of the solar neutrino experiments, as well as the SuperKamiokande zenith-angle distribution and the SuperKamiokande electron recoil energy spectrum.

\footnotetext{
${ }^{\dagger}$ Different physical mechanisms that might lead to an energy-independent supression may predict different zenith angle dependences for the detector event rates. Therefore, we have not included information about the zenith-angle distribution in the global fits for energy-independent supression.
} 
As described below in Sect. VIIIE, the best-fit global MSW solution for active neutrinos is: $\Delta m^{2}=5.4 \times 10^{-6} \mathrm{eV}^{2}, \sin ^{2} 2 \theta=5.5 \times 10^{-3}$ (and for sterile neutrinos: $\Delta m^{2}=4.0 \times$ $\left.10^{-6} \mathrm{eV}^{2}, \sin ^{2} 2 \theta=6.9 \times 10^{-3}\right)$. For vacuum oscillations, the best-fit solution is: $\Delta m^{2}=$ $6.5 \times 10^{-11} \mathrm{eV}^{2}, \sin ^{2} 2 \theta=0.75$.

\section{A. Indications of new physics}

The results from the first phase of the SuperKamiokande experiment have strengthened the inference that new physics is required to describe solar neutrino experiments.

If we consider only the total rates in the solar neutrino experiments, then there is no linear combination of the undistorted neutrino energy spectra that can fit the available data at the $3 \sigma$ level (see the discussion in Sec. [IB 1 and Sec. [IB 2). This result, whose physical basis is described in Sec. IIQ, is independent of any astrophysical arguments regarding the basic correctness of the solar model. In particular, the $3 \sigma$ discrepancy ignores the additional evidence provided by helioseismological measurements, which agree to high precision $(0.1 \%$ r.m.s in sound speeds, see Ref. [25]) with the predictions of the standard solar model. The Standard Solar Model predictions are inconsistent with the observed rates in solar neutrino experiments at approximately the $20 \sigma$ level (see Sec. ПA).

The data from the total rates alone indicate that the $\nu_{e}$ energy spectrum from the sun is distorted, i.e., the survival probability for electron type neutrinos to reach the earth is energy dependent.

If we impose the additional constraints from the measured SuperKamiokande spectrum and Day-Night asymmetry as well as the total rates, then an arbitrary linear combination of the $p p,{ }^{7} \mathrm{Be},{ }^{8} \mathrm{~B}$, and $\mathrm{CNO}$ neutrino fluxes is ruled out at the $3.5 \sigma$ confidence level (see Sec. VIIA). This minimum discrepancy again ignores all information about the solar model.

\section{B. Average event rates}

The most robust results of the solar neutrino experiments so far are the total observed rates, which are summarized in Table 1 . We have therefore evaluated accurately the allowed regions of neutrino parameters for either active or sterile MSW or vacuum neutrino oscillations, using the total rates as the only constraints. If neutrino oscillations are indeed occurring, a subset of the parameters that are consistent with the total rates must also be consistent with the other measured quantities (electron recoil energy spectrum and Day-Night asymmetry), a proposition that we have also tested.

The principal results considering only the total rates are displayed in Fig. 2-Fig. 8. We have also given in Sec. IV the best-fit neutrino oscillation parameters and mass differences for each scenario that we have discussed.

The most important change in the allowed range of neutrino parameters compared to our previous work 43] (which was prior to the announcement of the SuperKamiokande results) is that the SMA solution is shifted to somewhat smaller mixing angles, from the earlier value of $\sin ^{2} 2 \theta=8.7 \times 10^{-3}$ [43] to the current-fit value of $\sin ^{2} 2 \theta=6.0 \times 10^{-3}$ (Eq. 4a). The main causes of this shift are the smaller predicted ${ }^{8} \mathrm{~B}$ neutrino flux (for the INT normalization) and the the lower SuperKamiokande rate (somewhat lower than the Kamiokande rate). If 
the low energy cross section factor for the ${ }^{7} \mathrm{Be}(p, \gamma)^{8} \mathrm{~B}$ reaction is treated as a free parameter, then the best-fit mixing angle for the SMA solution decreases to an even smaller value of $\sin ^{2} 2 \theta=3.5 \times 10^{-3}$ (Eq. 12a). Further improvements in the most important nuclear cross sections are unlikely to reduce significantly the size of the allowed neutrino parameter regions (cf. Fig. 3), although further moderate shifts in the best-fit values may be anticipated.

The relatively modest shift in mixing angles from $\sin ^{2} 2 \theta=8.7 \times 10^{-3}$ to $\sin ^{2} 2 \theta=6.0 \times$ $10^{-3}$ has the effect of reducing drastically the predicted Day-Night asymmetry from what was expected earlier [43, i.e., 1.8\% for the best-fit SMA solution, to a probably un-measurably small $0.35 \%$ asymmetry for the current best-fit SMA solution (and is even smaller for the best-fit global solution, see Sect. VIIIE below). The Day-Night asymmetry is obviously sensitive to details of the neutrino parameter solutions.

Some theoretical models are already strongly disfavored by the constraints of the total rates alone. For example, models in which the suppression of electron type neutrinos is independent of energy are excluded at the $99.8 \%$ C. L. or more, depending upon the precise scenario considered (see the results described in Sec. IVD).

\section{Zenith-Angle dependence of rates}

No statistically significant Day-Night asymmetry or zenith-angle dependence of the solar neutrino event rate has been detected so far by the SuperKamiokande experiment [8 10]. The small-value of the observed Day-Night asymmetry excludes a large part of the LMA region in neutrino parameter space that is allowed if only the total solar neutrino rates are considered (see Fig. 10).

\section{Electron recoil energy spectrum}

The electron recoil energy distribution reported by SuperKamiokande [10] is inconsistent with no distortion at about the $99 \%$ C.L. . On the other hand, all of the popular neutrino oscillation solutions determined from the total rates only (SMA, LMA, and LOW MSW solutions and vacuum oscillations) provide acceptable, although not excellent, fits to the recoil energy spectrum (cf. Fig. 12). The observed distortion of the spectrum, i.e., the overall slope parameter, is in the direction predicted by the SMA solution. However, as emphasized by the SuperKamiokande Collaboration [10], the fits using the oscillation solutions for active neutrinos that are preferred by the total rates are not particularly good. The last three points in the recoil spectrum are somewhat higher than would have been expected from an undistorted ${ }^{8} \mathrm{~B}$ neutrino spectrum.

Figure 13 shows the regions of MSW parameter space that are allowed for both active and sterile neutrinos by imposing only the constraint of consistency with the electron recoil energy distribution. The best-fit solutions for the spectrum only, marked by dark points in Fig. 13, do not fall within the allowed regions determined by the global fit to all of the data (see Sec. VIIIE below). For vacuum oscillations, Fig. 14 shows the regions allowed by the spectral data alone. 


\section{E. Global analysis}

The combined constraints from the total rates, the zenith-angle dependence (or the DayNight Asymmetry), and the electron recoil energy spectrum provide the most comprehensive test of neutrino oscillation descriptions of solar neutrino experiments.

The allowed solution space for MSW oscillations, including the rates and the energy spectrum, is shown in Fig. 15a and the allowed solution space including all three sets of constraints, rates, energy spectrum, and zenith-angle dependence, is shown in Fig. 15b. The best-fit solution, in both cases, is close to the best-fit SMA solution when only the total rates are considered. The best-fit MSW solutions shown in both Fig. [15]a and Fig. [15]b are not particularly good, but are acceptable at the $7 \%$ C. L. . When all three sets of constraints are imposed, the SMA solution space is reduced somewhat in size compared to what is allowed if only the total rates are considered. For sterile neutrinos, the SMA solution is acceptable at the $8 \%$ C.L. ; the allowed region, which is similar to what is obtained for active neutrinos, is shown in Fig. 16. The LMA and LOW solutions are excluded for active and sterile neutrinos at the $99 \%$ C. L. when all three constraints, rates, spectrum, and Day-Night asymmetry, are included.

The global fits of MSW solutions to all the available data predict a Day-Night asymmetry, defined by Eq. (16), for the SuperKamiokande experiment (total energy $>6.5 \mathrm{MeV}$ ) that lies in the range $-0.0048<A<0.025$ for active neutrinos and $-0.0031<A<0.007$ for sterile neutrinos. The best-fit solutions predict an asymmetry whose absolute value is less than $0.1 \%$, un-measurably small, for both active and sterile neutrinos.

For vacuum oscillations, Fig. 17 shows the allowed solution space when the rates, energy spectrum, and zenith-angle dependence are all imposed. The best-fit vacuum solution is acceptable at the $6 \%$ C. L. . The inclusion of the Day-Night and the spectral constraints reduces considerably the parameter regions for vacuum oscillations (cf. Fig. 5).

Figure 18 shows how well (or poorly) the calculated global oscillation solutions fit the observed SuperKamiokande [10] electron recoil energy spectrum. Each of the three panels shows for a different global oscillation solution (described in Section VII) the ratio of the number of electrons in a given energy bin to the number expected using the undistorted neutrino energy spectrum [19] and the electroweak neutrino-electron scattering cross sections with radiative corrections [18]. The data shown were presented by the SuperKamiokande collaboration at Neutrino98 [10]. In computing the predictions for the different global oscillation solutions, we included the reported SuperKamiokande energy resolution and trigger efficiency function. All three of the best-fit global solutions fall well below the measured Ratio in the three highest energy bins. The possibility that this discrepancy at high energies might be due to a larger-than-expected cross section for the production of hep neutrinos is discussed extensively by Bahcall and Krastev [62]. The global best-fit vacuum oscillation solution shows a slight upturn at the lowest energies that might be detectable when SuperKamiokande obtains data with a threshold that extends to $5 \mathrm{MeV}$.

The neutrino oscillation predictions for the neutrino-electron scattering rate of the 0.86 $\mathrm{MeV}{ }^{7} \mathrm{Be}$ line, which will be measured by the BOREXINO neutrino experiment, are robust. Very similar predictions are obtained using only the total rates as a constraint and using the presently available data on the spectral shape and Day-Night asymmetry as well as the total rates. The results are summarized in Eq. (25), Eq. (26), and Eq. (27). 
We have also evaluated the globally allowed range of the $\nu_{e}$ survival probability which could be compared with future measurements of the $\nu_{e}{ }^{7} \mathrm{Be}$ neutrino flux, in a charged current (LENS) experiment. The results are given in Eq. (28), Eq. (29), and Eq. (30).

\section{F. Summation}

Different neutrino oscillation scenarios, including SMA MSW conversion to either active or sterile neutrinos and vacuum conversion to active neutrinos, give similar quality descriptions of the solar neutrino data, acceptable at the level 6\%-8\%. Moreover, with the strong evidence for atmospheric neutrino oscillations than is now available, an oscillation solution of the solar neutrino problem seems even more probable than before. In addition, any description of solar neutrinos that does not include some new physics that causes an energy dependence in the $\nu_{e}$ survival probability is strongly disfavored by the combined data from solar neutrino experiments, while standard solar model calculations accurately predict the helioseismologically measured sound velocities in the sun.

However, with the existing data it is not possible to determine which kind of transition(s) solar neutrinos undergo. Fits of neutrino oscillation scenarios to just the total event rates in the chlorine, Kamiokande, GALLEX, SAGE, and SuperKamiokande experiments, which may be the most robust currently available experimental information, suggest neutrino parameters in which the Day-Night asymmetry and the spectral distortion are difficult to measure. The situation may improve significantly when data from the lower-energy spectral bins of SuperKamiokande, at $5.5 \mathrm{MeV}$ and $6.0 \mathrm{MeV}$, are available. Crucial information will also be provided by the future SNO [13], BOREXINO [14], and GNO [63] experiments.

We suspect that the unique description of solar neutrino phenomena will require global analysis of all of the available data. We hope that this paper is a useful step along the path toward a complete solution of the solar neutrino problems.

\section{ACKNOWLEDGMENTS}

We are grateful to the chlorine, Kamiokande, GALLEX, SAGE, and SuperKamiokande collaborations for the superb data that made possible this analysis. We are especially grateful to the SuperKamiokande collaboration for making available preliminary data at Neutrino 98, in addition to the data in their formal paper [9], which permitted more stringent inferences. JNB acknowledges support from NSF grant No. PHY95-13835 and stimulating discussions with M. Cribier and R. Raghavan regarding the proposed LENS experiment. PIK acknowledges support from NSF grant No. PHY95-13835 and NSF grant No. PHY-9605140. AS thanks the Institute for Advanced Study for hospitality during a visit in which this work was initiated.

\section{APPENDIX: ANALYSIS DETAILS}

We describe here the methods used in our statistical analysis of the solar neutrino data. 


\section{Rates only}

In the analysis which includes only the event rates in the different detectors, we use the procedure described in detail in Ref. [34]. The $\chi^{2}$ for the combined fit is defined as:

$$
\left.\chi^{2} \text { (Rates }\right)=\Sigma_{i, j=1,4}\left(R_{i}^{\mathrm{th}}-R_{i}^{\mathrm{exp}}\right) V_{i, j}^{-1}\left(R_{j}^{\mathrm{th}}-R_{j}^{\exp }\right) .
$$

Here $R_{i}^{\text {th }}$ is the theoretically predicted event rate in the $i$-th detector (chlorine, SAGE, GALLEX and SuperKamiokande), which takes into account the electron neutrino survival probabilities, $R_{i}^{\exp }$ is the corresponding experimentally measured event rate, and $V_{i, j}$ is the error matrix which is a function of the theoretical uncertainties (nuclear cross-sections, age, luminosity and heavy element abundances in the standard solar model) as well as the experimental statistical and systematic errors from each experiment (see Table [I). The theoretical uncertainties are determined in BP98 [20]. Following BP98, we have included theoretical uncertainties due to the diffusion of elements in the sun and to the electron capture cross section on ${ }^{7} \mathrm{Be}$, not previously included as uncertainties in neutrino oscillation studies. A detailed description of how the individual uncertainties are calculated can be found at http://www.sns.ias.edu/ jnb/SNdata in the menu item 'Solar neutrino rates, fluxes, and uncertainties.' Important correlations exist between the neutrino fluxes that must be included correctly in the evaluation of the error matrix or the calculated allowed regions will be incorrectly reduced in size.

\section{Recoil electron spectrum}

Next we describe the analysis of the recoil electron spectrum in SuperKamiokande which includes a computation of the following $\chi^{2}$ function:

$$
\chi^{2}(\text { Spectrum })=\Sigma_{\mathrm{i}, \mathrm{j}=1,16}\left(\alpha \mathrm{S}_{\mathrm{i}}^{\text {th }}-\mathrm{S}_{\mathrm{i}}^{\exp }\right) \mathrm{W}_{\mathrm{i}, \mathrm{j}}^{-1}\left(\alpha \mathrm{S}_{\mathrm{j}}^{\text {th }}-\mathrm{S}_{\mathrm{j}}^{\exp }\right) .
$$

Here $S_{i}^{\text {th }}$ is the theoretically predicted event rate for the $i$-th energy bin in SuperKamiokande and $S^{\exp }$ is the corresponding experimentally measured event rate. In this paper, we use the measured electron recoil energy spectrum data presented by the SuperKamiokande Collaboration [10] at Neutrino 98 and the recently determined undistorted spectrum [19]. The statistical as well as the systematic experimental errors are included. Since the latter are fully correlated, we assume a correlation coefficient of 1 between each pair of bins. Neglect of these correlations would lead to an appreciable increase of the regions that is excluded. The entries in the covariance matrix are:

$$
W_{i, j}=\sigma_{i}^{\text {stat }} \sigma_{j}^{\text {stat }} \delta_{i, j}+\sigma_{i}^{\text {syst }} \sigma_{j}^{\text {syst }} .
$$

The coefficient $\alpha$ in Eq. (A2) is an overall normalization coefficient and is varied as a free parameter independent of $\Delta m^{2}$ and $\sin ^{2} 2 \theta$. This variation reflects the fact that we are interested here in a test of the shape of the measured spectrum and not in the overall event rate in SuperKamiokande.

We calculate the theoretically expected event rates in the individual bins including the quoted energy resolution and trigger efficiency function in SuperKamiokande. We neglect the uncertainty in the energy normalization since, according to the SuperKamiokande Collaboration, it is less than $1 \%$. 


\section{Day-Night asymmetry and zenith angular dependence}

The Day-Night asymmetry measured by SuperKamiokande [10] is not significantly different from zero, which limits the allowed neutrino oscillation parameters. Assuming $3 \sigma$ errors and combining the statistical and systematic errors in quadrature, the asymmetry $A_{\text {exp }}$, defined by Eq. (16), is

$$
-0.096<A_{\exp }<0.050 .
$$

The lower limit in Eq. (A4) is not especially useful since we have found by explicit calculation that asymmetries predicted by MSW parameters in the entire range $10^{-9} \mathrm{eV}^{2}<$ $\Delta m^{2}<10^{-3} \mathrm{eV}^{2}$ and $10^{-4}<\sin ^{2} 2 \theta<1.0$, never take on very large negative values $(A>$ $-0.005)$. However, the upper limit is exceeded for certain choices of previously-allowed oscillation parameters; these parameters are therefore excluded by the SuperKamiokande measurement.

Since the significant theoretical uncertainties cancel in the ratio, we define $\chi^{2}$ for the Day-Night asymmetry as:

$$
\chi^{2}(D / N)=\left(A_{\text {th }}-A_{\exp }\right) / \Delta A_{\exp },
$$

where $A_{\text {th }}$ is the theoretically predicted asymmetry due to neutrino regeneration in the earth (zero for vacuum oscillations) and $\Delta A_{\exp }=0.024$ is the combined statistical plus systematic error given by the SuperKamiokande Collaboration.

Figure 10 and Figure 11 show the excluded regions for active and sterile neutrinos, respectively. Note that the relatively large uncertainties in the total ${ }^{8} \mathrm{~B}$ neutrino flux cancel in forming the asymmetry ratio and therefore do not affect the excluded regions. Also, we have verified that at the present level of accuracy the excluded regions are not affected significantly by the uncertainty in the undistorted ${ }^{8} \mathrm{~B}$ neutrino spectrum.

The measured zenith angular distribution provides another constraint on neutrino oscillation parameters. We have calculated the predicted zenith angular distribution of events for many points in the $\sin ^{2} 2 \theta-\Delta m^{2}$ plane and have compared the predicted distributions with the binned zenith-angle distribution presented [10] by the SuperKamiokande Collaboration at the Neutrino 98 conference.

The $\chi^{2}$ in this case is defined by

$$
\chi^{2}(\text { Zenith })=\Sigma_{\mathrm{i}, \mathrm{j}=1,10}\left(\alpha \mathrm{Z}_{\mathrm{i}}^{\text {th }}-\mathrm{Z}_{\mathrm{i}}^{\exp }\right) \mathrm{U}_{\mathrm{i}, \mathrm{j}}^{-1}\left(\alpha \mathrm{Z}_{\mathrm{j}}^{\text {th }}-\mathrm{Z}_{\mathrm{j}}^{\exp }\right) .
$$

The predicted distribution for the zenith angle dependence was calculated by assuming uninterrupted operation of the SuperKamiokande detector during 504 days starting from April 1, 1996. This assumption is not precisely correct since the detector has been occasionally shut down for calibration and maintenance. However, since these interruptions are a small fraction of the data taking period, our calculation is not seriously degraded by the lack of published information about the detailed operating schedule.

The SuperKamiokande Collaboration can perform a unique public service by testing the sensitivity of their inferences based upon the zenith angle dependence to the assumptions regarding the operating schedule. They can test at what quantitative level the small effects due to the precise operating schedule affect the conclusions regarding neutrino parameters. 
The matrix $U$ that appears in Eq. (A6) is assumed, in our calculations, to be diagonal. Correlations between the 10 bins of the zenith angular distribution might conceivably arise from systematic effects (for example, electrons falling in different bins being detected by the same photomultiplier tubes). Although these correlations are expected to be small, it would be very useful if the SuperKamiokande Collaboration were to publish their estimates of any correlations that cause $U$ to be non-diagonal.

\section{Straight line fit}

As discussed in Sec. VIG, the ratio, $R$, of the measured to the expected spectral energy distribution can be fit by a straight line, as in Eq. (17. For an undistorted spectrum, $R$ is a constant independent of energy.

The $\chi^{2}$ in this case is defined as a sum over the 16 energy bins of the electron spectrum:

$$
\chi^{2}(\text { Line })=\Sigma_{\mathrm{i}, \mathrm{j}=1,16}\left(\alpha \mathrm{L}_{\mathrm{i}}^{\text {th }}-\mathrm{S}_{\mathrm{i}}^{\text {exp }}\right) \mathrm{W}_{\mathrm{i}, \mathrm{j}}^{-1}\left(\alpha \mathrm{L}_{\mathrm{j}}^{\text {th }}-\mathrm{S}_{\mathrm{j}}^{\exp }\right),
$$

where $L_{i}^{\text {th }}=R_{0}+S_{0}\left(E_{e, i}-E_{0}\right), E_{0}=10 \mathrm{MeV}$ is a conveniently-chosen energy, $E_{e, i}$ is the total electron energy in the i-th bin (we use the energy in the middle of each energy bin), and $S_{0}$ and $R_{0}$ are arbitrary parameters which are varied until the minimum $\chi^{2}$ is found. The covariance matrix $W$ is the same as the covariance matrix defined in Eq. (A2).

\section{Global fits}

In Sec. VII, we combine in different ways the constraints from the measured total rates, the spectrum shape, and the Day-Night asymmetry (or zenith-angle dependence). In each case, we add the $\chi^{2}$ for each data set ( rates, energy spectrum, and Day-Night asymmetry or zenith-angle distribution). The individual $\chi^{2}$ are defined in previous sections of this appendix and are treated as independent in the global fits. Thus in Sec. VTT the effective $\chi^{2}$ are always the sum of two or three independent terms. 


\section{REFERENCES}

[1] R. Davis, Jr., D. S. Harmer, and K. C. Hoffman, Phys. Rev. Lett. 20, 1205 (1968).

[2] J. N. Bahcall, N. A. Bahcall, and G. Shaviv, Phys. Rev. Lett. 20, 1209 (1968).

[3] J. N. Bahcall and R. Davis, Jr., Science 191, 264 (1976).

[4] J. N. Bahcall, Neutrino Astrophysics (Cambridge University Press, Cambridge, England, 1989).

[5] KAMIOKANDE Collaboration, Y. Fukuda et al., Phys. Rev. Lett. 77, 1683 (1996).

[6] GALLEX Collaboration, P. Anselmann et al., Phys. Lett. B 342, 440 (1995); GALLEX Collaboration, W. Hampel et al., Phys. Lett. B 388, 364 (1996).

[7] SAGE Collaboration, V. Gavrin et al., in Neutrino 96, Proceedings of the XVII International Conference on Neutrino Physics and Astrophysics, Helsinki, edited by K. Huitu, K. Enqvist and J. Maalampi (World Scientific, Singapore, 1997), p. 14; V. Gavrin et al., in Neutrino 98, Proceedings of the XVIII International Conference on Neutrino Physics and Astrophysics, Takayama, Japan, 4-9 June 1998, edited by Y. Suzuki and Y. Totsuka. To be published in Nucl. Phys. B (Proc. Suppl.).

[8] SuperKamiokande Collaboration, Y. Fukuda et al. Phys. Rev. Lett. (accepted for publication, hep-ex/9805021).

[9] R. Svoboda (for the SuperKamiokande Collaboration), ITP Conference on Solar neutrinos: News About SNUs, December 2-6, 1997 (unpublished), http://www.itp.ucsb.edu/online/snu/svoboda/; H. Sobel (for the SuperKamiokande Collaboration), Aspen Winter Conference on Particle Physics, January 25-31, 1998, Aspen, CO (unpublished). http://www.physics.arizona.edu ina/aspen98.html.

[10] SuperKamiokande Collaboration, Y. Suzuki, in Neutrino 98, Proceedings of the XVIII International Conference on Neutrino Physics and Astrophysics, Takayama, Japan, 4-9 June 1998, edited by Y. Suzuki and Y. Totsuka. To be published in Nucl. Phys. B (Proc. Suppl.).

[11] L. Wolfenstein, Phys. Rev. D 17, 2369 (1978); S. P. Mikheyev and A. Yu. Smirnov, Yad. Fiz. 42, 1441 (1985) [Sov. J. Nucl. Phys. 42, 913 (1985)]; Nuovo Cimento C 9, 17 (1986).

[12] V. N. Gribov and B. M. Pontecorvo, Phys. Lett. B 28, 493 (1969).

[13] A. B. McDonald, in Proceedings of the 9th Lake Louise Winter Institute, edited by A. Astbury et al. (World Scientific, 1994), p. 1.

[14] C. Arpesella et al., BOREXINO proposal, Vols. 1 and 2, eds. G. Bellini, R. Raghavan et al. (Univ. of Milano, Milano, 1992).

[15] J. N. Bahcall, Phys. Rev. C 56, 3391 (1997).

[16] J. N. Bahcall et al., Phys. Rev. C 54, 411 (1996).

[17] J. N. Bahcall and R. Ulrich, Rev. Mod. Phys. 60, 297 (1988).

[18] J. N. Bahcall, M. Kamionkowski, and A. Sirlin, Phys. Rev. D 51, 6146 (1995).

[19] J. N. Bahcall, E. Lisi, D. E. Alburger, L. De Braeckeleer, S. J. Freedman, and J. Napolitano, Phys. Rev. C 54, 411 (1996).

[20] J. N. Bahcall, S. Basu, and M. H. Pinsonneault, to be published in Phys. Lett. B, July 1998, astro-ph/9805135.

[21] E. Adelberger et al., Rev. Mod. Phys. (to be published, October 1998).

[22] C. W. Johnson, E. Kolbe, S. E. Koonin, and K. Langanke, Astrophys. J. 392, 320 (1992). 
[23] S. M. Bilenky and C. Giunti, "Implications of CHOOZ Results for the Decoupling of Solar and Atmospheric Neutrino Oscillations," hep-ph/9802201.

[24] CHOOZ experiment: M. Apollonio et al., Phys. Lett. B 420, 397 (1998).

[25] J. N. Bahcall, M. H. Pinsonneault, S. Basu, and J. Christensen-Dalsgaard, Phys. Rev. Lett. 78, 171 (1997).

[26] J. N. Bahcall and M. H. Pinsonneault, Rev. Mod. Phys. 64, 885 (1992).

[27] J. N. Bahcall and M. H. Pinsonneault, Rev. Mod. Phys. 67, 781 (1995).

[28] N. Hata, S. Bludman, and P. Langacker, Phys Rev D 49, 3622 (1994).

[29] S. Parke, Phys. Rev. Lett. 74, 839 (1995).

[30] K. M. Heeger and R. G. H. Robertson, Phys Rev Lett 77, 3720 (1996).

[31] J. N. Bahcall and P. I. Krastev, Phys. Rev. D 53, 4211 (1996).

[32] J. N. Bahcall and H. A. Bethe, Phys. Rev. Lett. 65, 2233 (1990).

[33] N. Hata and P. Langacker, Phys. Rev. D 52, 420 (1995).

[34] G. L. Fogli, E. Lisi, and D. Montanino, Phys. Rev. D 49, 3226 (1994); see also G. L. Fogli and E. Lisi, Astropart. Phys. 3, 185 (1995).

[35] V. Berezinsky, G. Fiorentini, and M. Lissia, Phys. Lett. B 341, 38 (1995).

[36] N. Hata and P. Langacker, Phys. Rev. D 50, 632 (1993).

[37] P. Krastev and S. T. Petcov, Phys. Rev. Lett. 72, 1960 (1994); Nucl. Phys. B 449, 605 (1995).

[38] E. Gates, L. M. Krauss, and M. White, Phys. Rev. D 51, 2631 (1995).

[39] T. K. Kuo and J. Pantaleone, Rev. Mod. Phys. 61, 937 (1987).

[40] E. Calabresu, N. Ferrari, G. Fiorentini, and M. Lissia, preprint INFN-FE-10-95, June 1995, hep-ph/9507352 (unpublished).

[41] M. Maris and S. T. Petcov, Phys. Rev. D 56, 7444 (1997).

[42] Z. Berezhiani and A. Rossi, Phys. Rev. D 51, 5229 (1995).

[43] J. N. Bahcall and P. I. Krastev, Phys. Rev. C 56, 2839 (1997).

[44] P. Krastev and S. T. Petcov, Phys. Lett. B 207, 64 (1988); [Erratum: B214, 661 (1988)].

See also S. T. Petcov, Phys. Lett. B 200, 373 (1988); 214, 139 (1988).

[45] B. T. Cleveland et al., Astrophys. J. 496, 505 (1998); B. T. Cleveland et al., Nucl. Phys. B (Proc. Suppl.) 38, 47 (1995); R. Davis, Prog. Part. Nucl. Phys. 32, 13 (1994).

[46] P. I. Krastev and S. T. Petcov, Phys. Lett. B 299, 99 (1993); G. L. Fogli, E. Lisi, and D. Montanino, Phys. Rev. D 54, 2048 (1996).

[47] A. J. Baltz and J. Weneser, Phys. Rev. D 50, 5971 (1994); 51, 3960 (1995).

[48] P. Krastev and A. Smirnov, Phys. Lett. B 338, 282 (1994).

[49] P. Krastev and S. Petcov, Phys. Rev. D 53, 1665 (1996).

[50] S. Nussinov, Phys. Lett. B 63, 201 (1976).

[51] P. F. Harrison, D. H. Perkins, and W. G. Scott, Phys. Lett. B 349, 137 (1995); 396, 186 (1997).

[52] R. Foot and R. R. Volkas, Phys. Rev. D 52, 6595 (1995).

[53] G. Comforto, A. Marchionni, F. N. Martelli, F. Vetrano, M. Lanfranchi, and G. Torricelli-Ciamponi, Astropart. Phys. 5, 147 (1996).

[54] A. Acker and S. Pakvasa, Phys. Lett. B 397, 209 (1997); A. Acker, S. Pakvasa, J. Leaned and T. Weiler, Phys. Lett. B 298, 149 (1993).

[55] P. I. Krastev and S. T. Petcov, Phys. Lett. B 395, 69 (1997).

[56] S. P. Mikheyev and A. Yu. Smirnov, in '86 Massive Neutrinos in Astrophysics and in 
Particle Physics, Proceedings of the Sixth Moriond Workshop, edited by O. Fackler and Y. Trân Thanh Vân (Editions Frontières, Gif-sur-Yvette, 1986), p. 355; J. Bouchez et al., Z. Phys. C 32, 499 (1986); M. Cribier, W. Hampel, J. Rich, and D. Vignaud, Phys. Lett. B 182, 89 (1986); M. L. Cherry and K. Lande, Phys. Rev. D 36, 3571 (1987); S. Hiroi, H. Sakuma, T. Yanagida, and M. Yoshimura, Phys. Lett. B 198, 403 (1987); S. Hiroi, H. Sakuma, T. Yanagida, and M. Yoshimura, Prog. Theor. Phys. 78, 1428 (1987); A. Dar, A. Mann, Y. Melina, and D. Zajfman, Phys. Rev. D 35, 3607 (1988); M. Spiro and D. Vignaud, Phys. Lett. B 242, 279 (1990).

[57] A. J. Baltz and J. Weneser, Phys. Rev. D 35, 528 (1987).

[58] A. J. Baltz and J. Weneser, Phys. Rev. D 37, 3364 (1988).

[59] J. N. Bahcall, Phys. Rev. D 44, 1644 (1991).

[60] W. Kwong and S. P. Rosen, Phys. Rev. Lett. 68, 748 (1992); P. I. Krastev and A. Yu. Smirnov, Phys. Lett. B 338, 282 (1994); W. Kwong and S. P. Rosen, Phys. Rev. D 44, 2241 (1995); P. I. Krastev and S. T. Petcov, Nucl. Phys. B 449, 605 (1995).

[61] R. S. Raghavan, Phys. Rev. Lett. 78, 3618 (1997).

[62] J. N. Bahcall and P. Krastev, Phys. Lett. B (in press) (1998), hep-ph/9807525.

[63] E. Bellotti et al., "Proposal for a Permanent Gallium Neutrino Observatory (GNO) at Laboratori Nazionali Del Gran Sasso," http://kosmopc.mpihd.mpg.de/gallex/gallex.html, February (1996).

[64] (GONG) J. Christensen-Dalsgaard et al., GONG Collaboration, Science 272, 1286 (1996); (BP95) J. N. Bahcall and M. H. Pinsonneault, Rev. Mod. Phys. 67, 781 (1995); (KS94) A. Kovetz and G. Shaviv, Astrophys. J. 426, 787 (1994); (CDF94) V. Castellani, S. Degl'Innocenti, G. Fiorentini, L. M. Lissia, and B. Ricci, Phys. Lett. B 324, 425 (1994); (JCD94) J. Christensen-Dalsgaard, Europhys. News 25, 71 (1994); (SSD94) X. Shi, D. N. Schramm, and D. S. P. Dearborn, Phys. Rev. D 50, 2414 (1994); (DS96) A. Dar and G. Shaviv, Astrophys. J. 468, 933 (1996); (CDF93) V. Castellani, S. Degl'Innocenti, and G. Fiorentini, Astron. Astrophys. 271, 601 (1993); (TCL93) S. Turck-Chièze and I. Lopes, Astrophys. J. 408, 347 (1993); (BPML93) G. Berthomieu, J. Provost, P. Morel, and Y. Lebreton, Astron. Astrophys. 268, 775 (1993); (BP92) J. N. Bahcall and M. H. Pinsonneault, Rev. Mod. Phys. 64, 885 (1992); (SBF90) I.-J. Sackman, A. I. Boothroyd, and W. A. Fowler, Astrophys. J. 360, 727 (1990); (BU88) J. N. Bahcall and R. K. Ulrich, Rev. Mod. Phys. 60, 297 (1988); (RVCD96) O. Richard, S. Vauclair, C. Charbonnel, and W. A. Dziembowski, Astron. Astrophys. 312, 1000 (1996); (CDR97) F. Ciacio, S. Degl'Innocenti, and B. Ricci, Astron. Astrophys. Suppl. Ser. 123, 449 (1997). 


\section{TABLES}

TABLE I. Solar neutrino data used in the analysis. The experimental results are given in SNU for all of the experiments except Kamiokande (and SuperKamiokande), for which the result is expressed as the measured ${ }^{8} \mathrm{~B}$ flux above $7.5 \mathrm{MeV}(6.5 \mathrm{MeV})$ in units of $10^{6} \mathrm{~cm}^{-2} \mathrm{~s}^{-1}$ at the earth. The ratios of the measured values to the corresponding predictions in the Bahcall-Pinsonneault standard solar model (BP98) of Ref. [20] are also given. The INT normalization (or, in parentheses, the CIT normalization) is used in these calculations. Only experimental errors are included in the column labeled Result/Theory. The results cited for the Kamiokande and SuperKamiokande experiments assume that the shape of the ${ }^{8} \mathrm{~B}$ neutrino spectrum is not affected by physics beyond the standard electroweak model.

\begin{tabular}{lcccccc}
\hline \hline Experiment & Result & \multicolumn{2}{c}{ Theory } & \multicolumn{2}{c}{ Result/Theory } & \multicolumn{2}{c}{ Reference } \\
\hline Homestake & $2.56 \pm 0.16 \pm 0.16$ & $7.7_{-1.0}^{+1.2}$ & $\left(8.8_{-1.1}^{+1.4}\right)$ & $0.33 \pm 0.029$ & $(0.29)$ & [45] \\
Kamiokande & $2.80 \pm 0.19 \pm 0.33$ & $5.15_{-0.7}^{+1.0}$ & $\left(6.1_{-0.9}^{+1.1}\right)$ & $0.54 \pm 0.07$ & $(0.46)$ & {$[5]$} \\
GALLEX & $77.5 \pm 6.2_{-4.7}^{+4.3}$ & $129_{-6}^{+8}$ & $\left(131_{-7}^{+9}\right)$ & $0.60 \pm 0.06$ & $(0.59)$ & {$[6]$} \\
SAGE & $66.6_{-8.1}^{+7.8}$ & $129_{-6}^{+8}$ & $\left(131_{-7}^{+9}\right)$ & $0.52 \pm 0.06$ & $(0.51)$ & {$[7]$} \\
SuperKamiokande & $2.44 \pm+0.05_{-0.07}^{+0.09}$ & $5.15_{-0.7}^{+1.0}$ & $\left(6.1_{-0.9}^{+1.1}\right)$ & $0.474 \pm 0.020$ & $(0.39)$ & \\
\end{tabular}

TABLE II. Minimum $\chi^{2}$ for different neutrino oscillation solutions of the solar neutrino problems The reference solar model corresponds to the INT normalization (or, in parentheses, the CIT normalization) of $S_{17}(0)$. Results are given for oscillations into either either active or sterile neutrinos.

\begin{tabular}{lcccc}
\hline \hline Solution & \multicolumn{3}{c}{ MSW } & Vacuum Oscillations \\
\hline active & SMA & LMA & LOW & \\
\hline sterile & $1.7(2.8)$ & $4.3(1.9)$ & $7.4(8.2)$ & $4.3(2.7)$ \\
\hline \hline
\end{tabular}




\section{FIGURES}

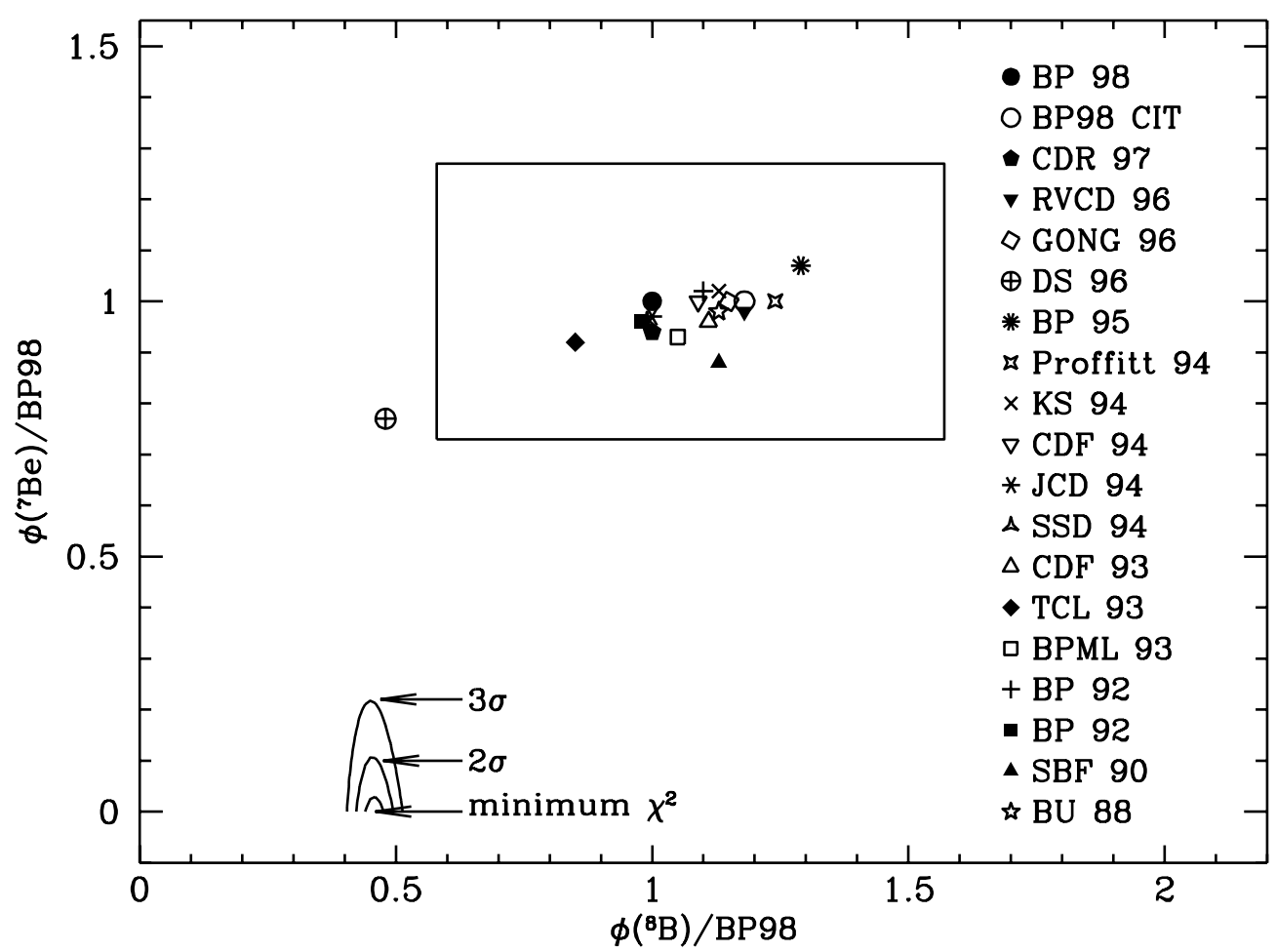

FIG. 1. Predictions of standard solar models since 1988. The figure shows the predictions of 19 standard solar models in the plane defined by the ${ }^{7} \mathrm{Be}$ and ${ }^{8} \mathrm{~B}$ neutrino fluxes. The abbreviations that are used in the figure to identify different solar models are defined in the bibliographical item, Ref. [64. We include all standard solar models with which we are familiar that were published in refereed journals in the decade 1988-1998. All of the fluxes are normalized to the predictions of the Bahcall-Pinsonneault 98 solar model, BP98 [20]. The rectangular error box defines the $3 \sigma$ error range of the BP98 fluxes. The best-fit ${ }^{7} \mathrm{Be}$ neutrino flux is negative. At the $99 \%$ C.L., there is no solution with all positive neutrino fluxes if the fluxes of CNO neutrinos are arbitrarily set equal to zero. There is no solution at the $99.9 \%$ C.L. if the CNO neutrinos are fixed at their standard solar model values. All of the standard model solutions lie far from the best-fit solution, even far from the $3 \sigma$ contour. 


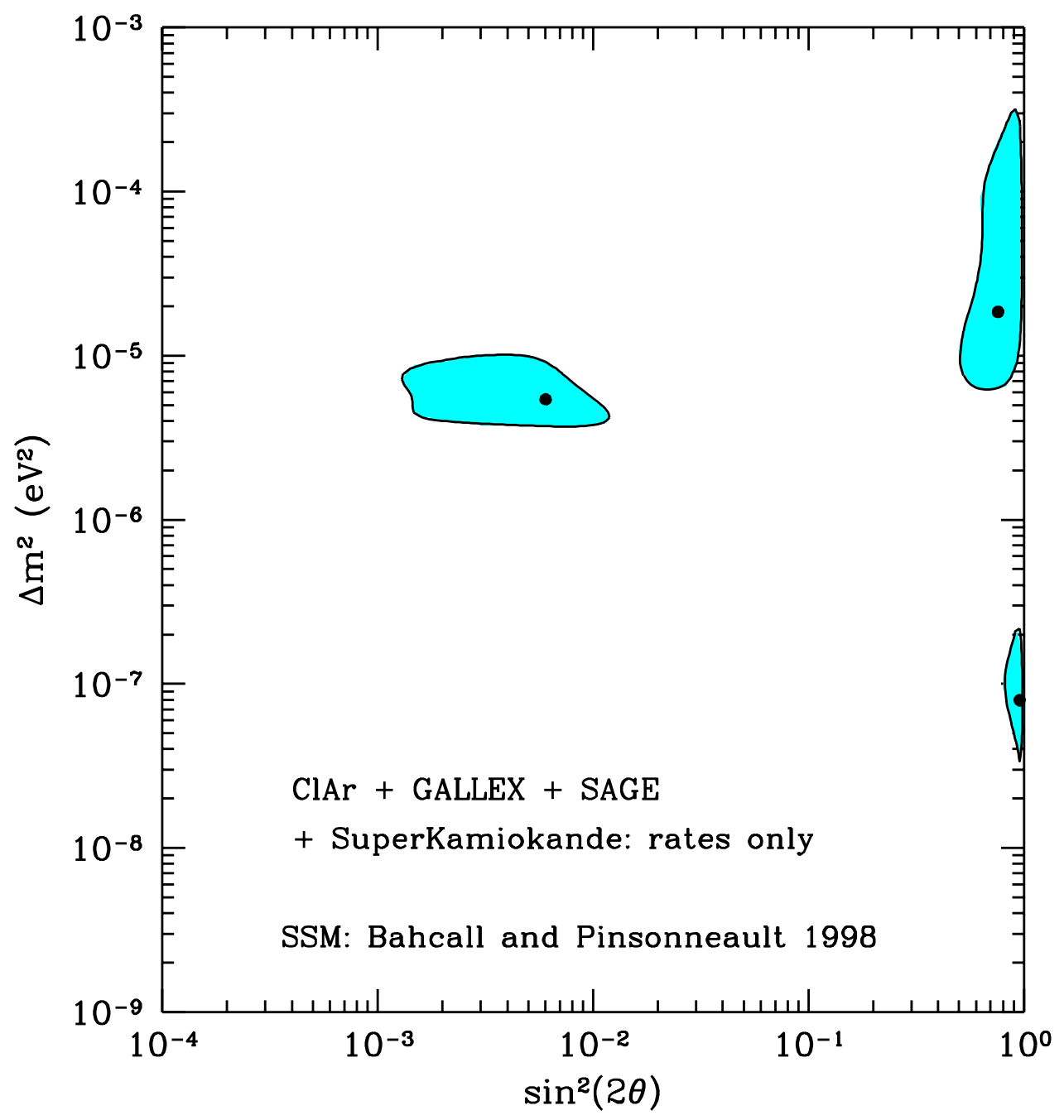

FIG. 2. MSW solutions: rates only. The figure shows the allowed regions (99\% C.L.) in $\Delta m^{2}$ - $\sin ^{2} 2 \theta$ parameter space for the MSW solution. The best fit points are indicated by dark circles. Only the total event rates in the chlorine, SuperKamiokande, GALLEX, and SAGE experiments are considered; the solar neutrino data are summarized in Table 1 . The ${ }^{8} \mathrm{~B}$ neutrino flux corresponds to the "INT normalization" of $\mathrm{S}_{17}(0)$. The neutrino transitions in the sun are assumed to be between active neutrinos $\left(\nu_{e} \rightarrow \nu_{\mu}\right.$ or $\left.\nu_{e} \rightarrow \nu_{\tau}\right)$. 

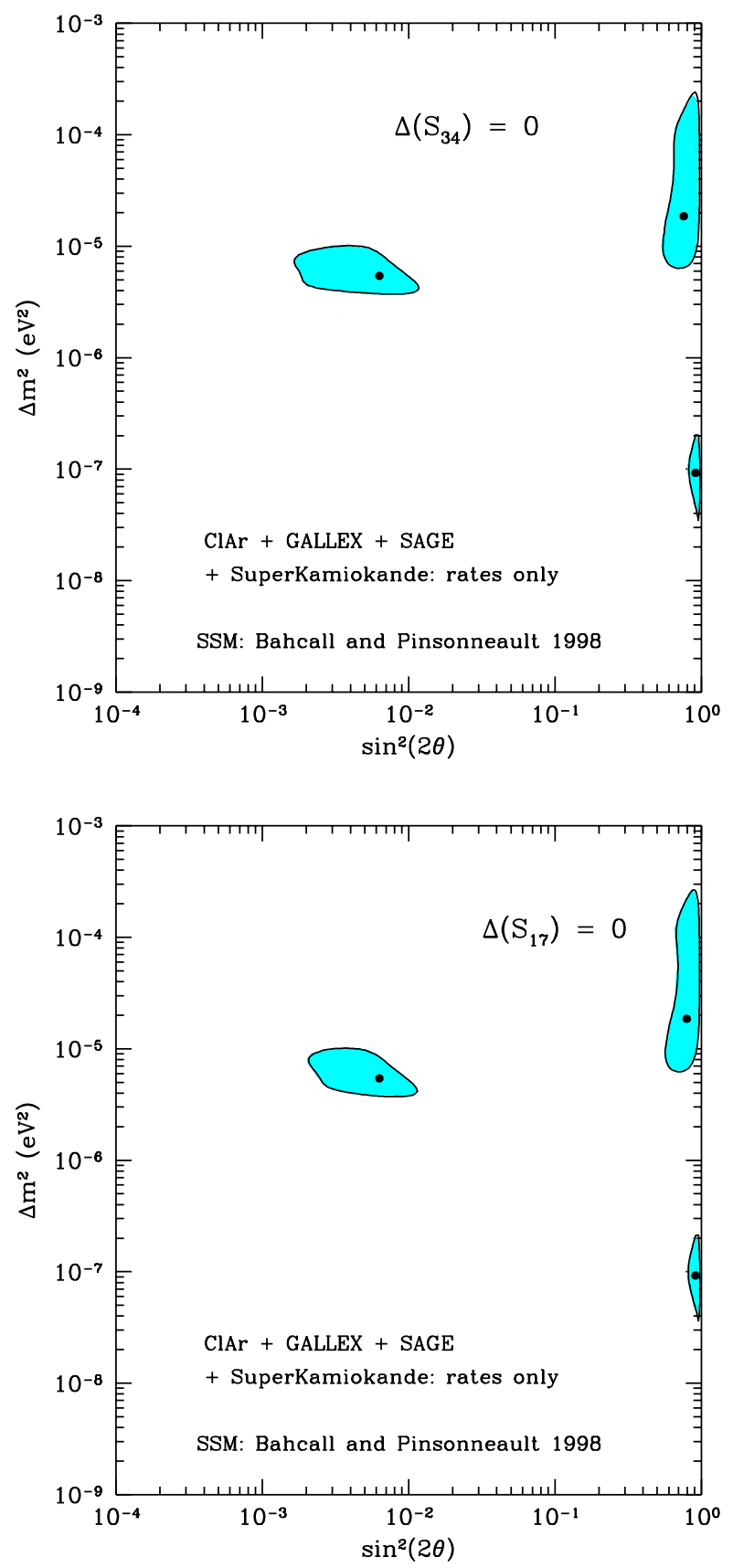

FIG. 3. Effect of uncertainties in $S_{34}$ and $S_{17}$ on the allowed MSW solution space. The panels are the same as in Fig. 2 except that in the top panel the uncertainty in $S_{34}$ is set equal to zero and in the lower panel the uncertainty in $S_{17}$ is set equal to zero. There is no large reduction in the MSW allowed regions if either $S_{34}$ or $S_{17}$ is presumed to be negligible. 


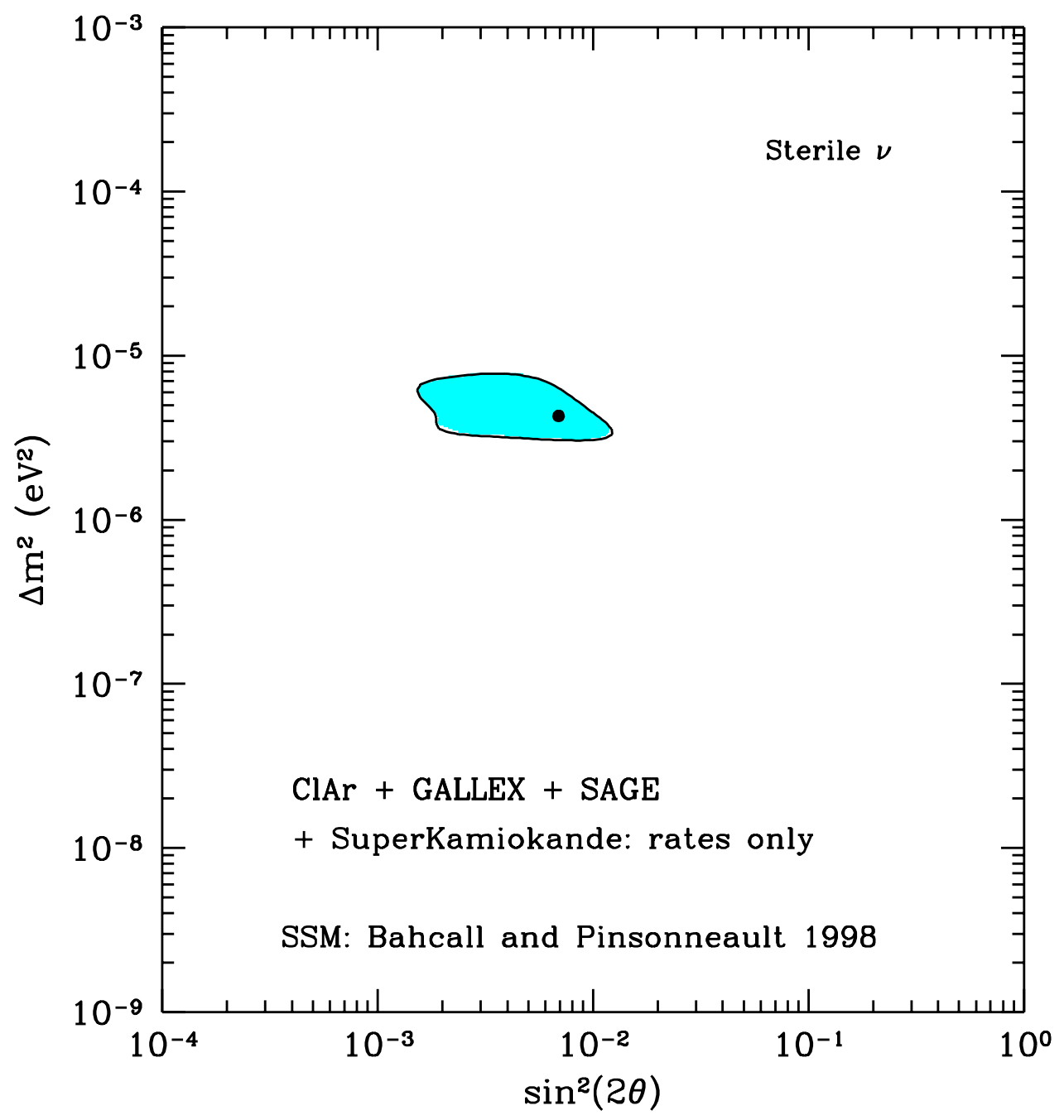

FIG. 4. Sterile neutrinos: rates only. The figure shows the regions in $\Delta m^{2}-\sin ^{2} 2 \theta$ parameter space that the total rates in the chlorine, SuperKamiokande, GALLEX, and SAGE experiments allow for MSW oscillations between an electron type neutrino and a sterile neutrino $\left(\nu_{e} \rightarrow \nu_{s}\right)$. Other conditions are the same as for Figure 2 . 


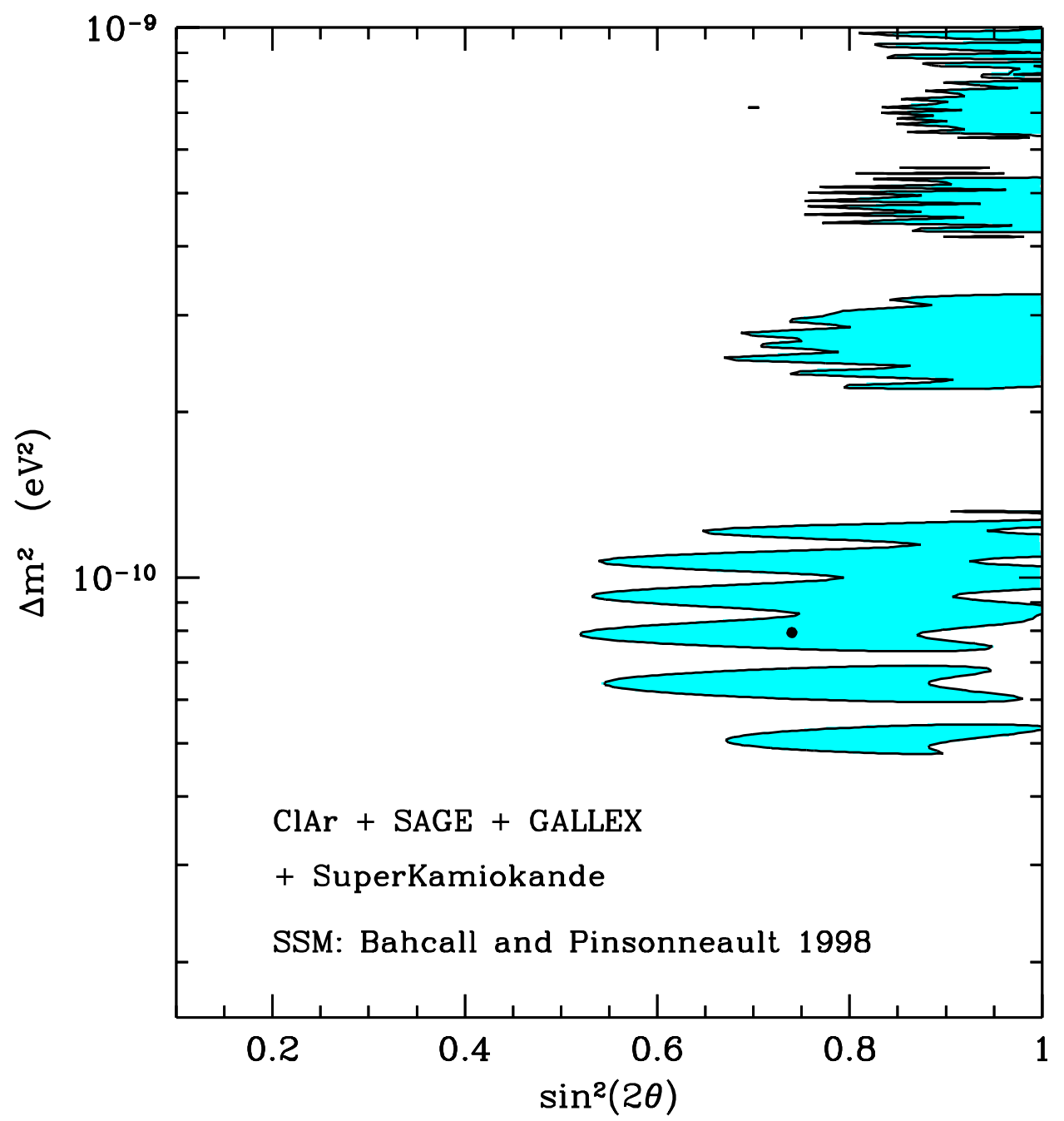

FIG. 5. Vacuum oscillations: rates only. The figure shows the regions in $\Delta m^{2}-\sin ^{2} 2 \theta$ parameter space that the total rates in the chlorine, SuperKamiokande, GALLEX, and SAGE experiments allow for vacuum neutrino oscillations between active neutrinos. The best-fit point is indicated by a dark circle. The experimental rates are summarized in Table 1 . 


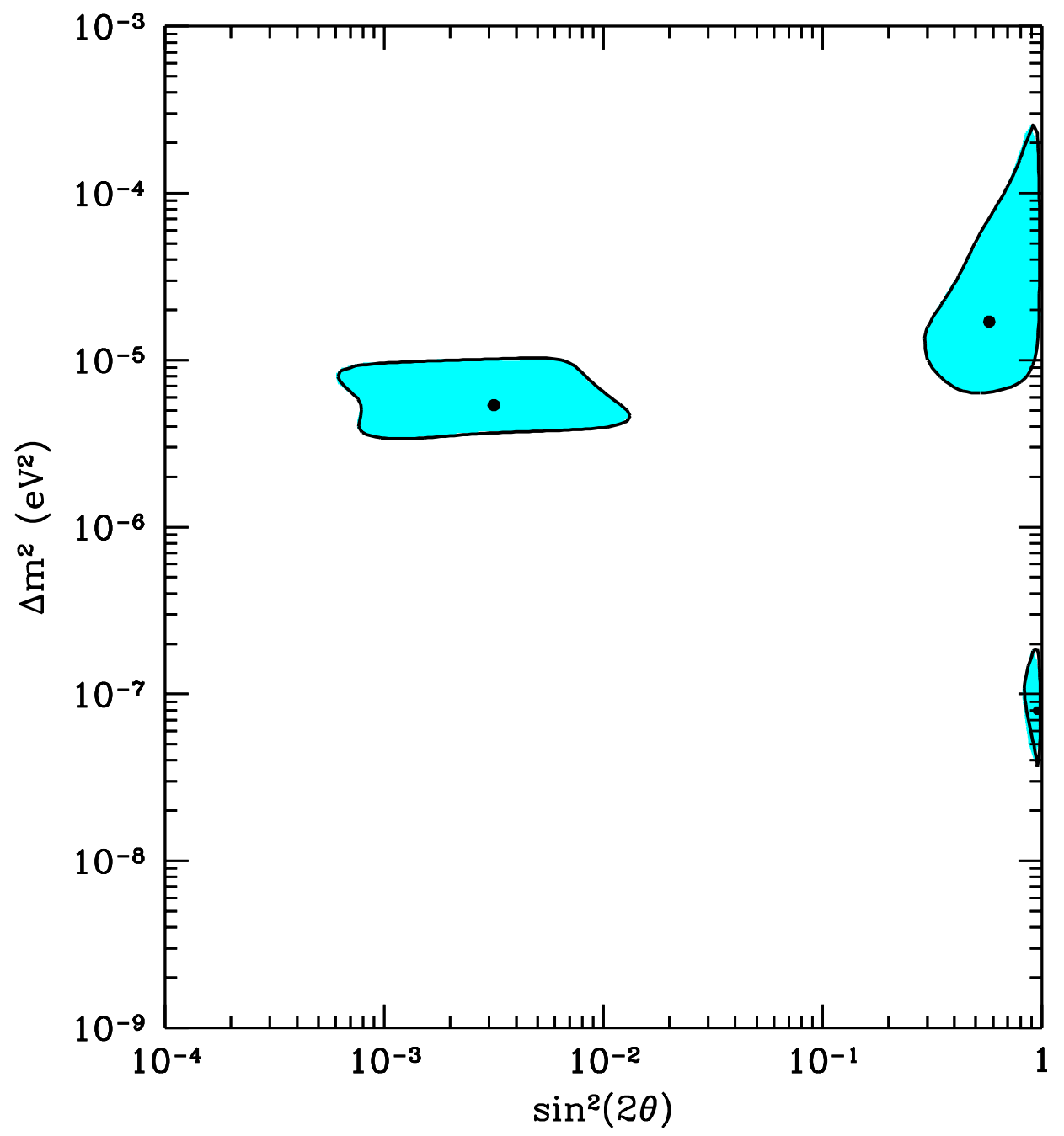

FIG. 6. Variable ${ }^{8} \mathrm{~B}$ flux and MSW solutions. The figure shows the allowed regions at $99 \%$ C.L. in the $\Delta m^{2}-\sin ^{2} 2 \theta$ plane for the MSW solutions with an arbitrary ${ }^{8} \mathrm{~B}$ neutrino flux (treated as a free parameter). 


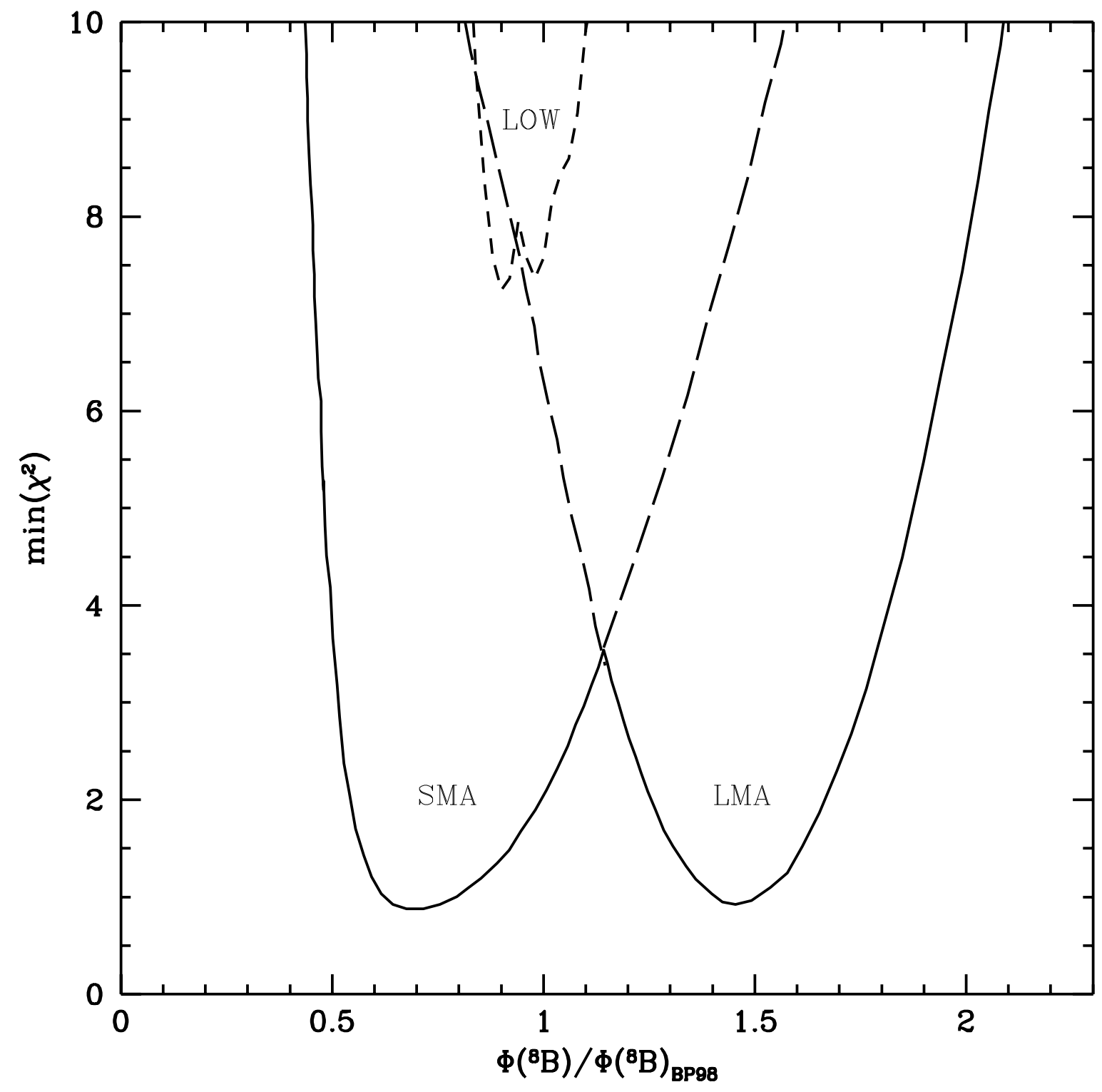

FIG. 7. Minimum $\chi^{2}$ (MSW solution) as a function of the boron neutrino flux. The oscillations are between active neutrinos $\left(\nu_{e} \rightarrow \nu_{\mu}\right.$ or $\left.\nu_{e} \rightarrow \nu_{\tau}\right)$. The reference boron flux in the BP98 solar model corresponds to INT normalization of $S_{17}(0)$. 


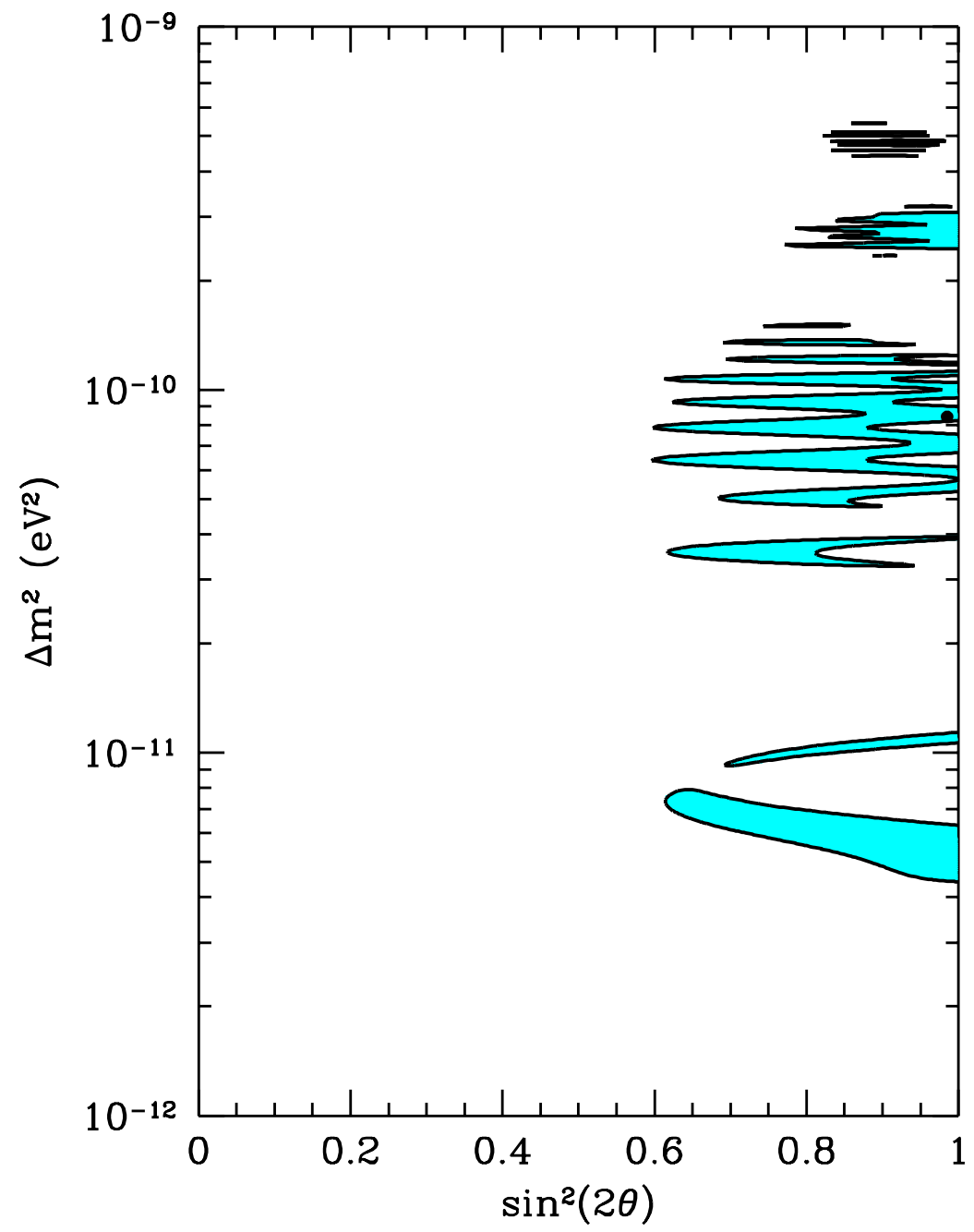

FIG. 8. Allowed regions in $\Delta m^{2}-\sin ^{2} 2 \theta$ parameter space for vacuum oscillations with an arbitrary ${ }^{8} \mathrm{~B}$ neutrino flux. The oscillations are assumed to occur between active neutrinos. 


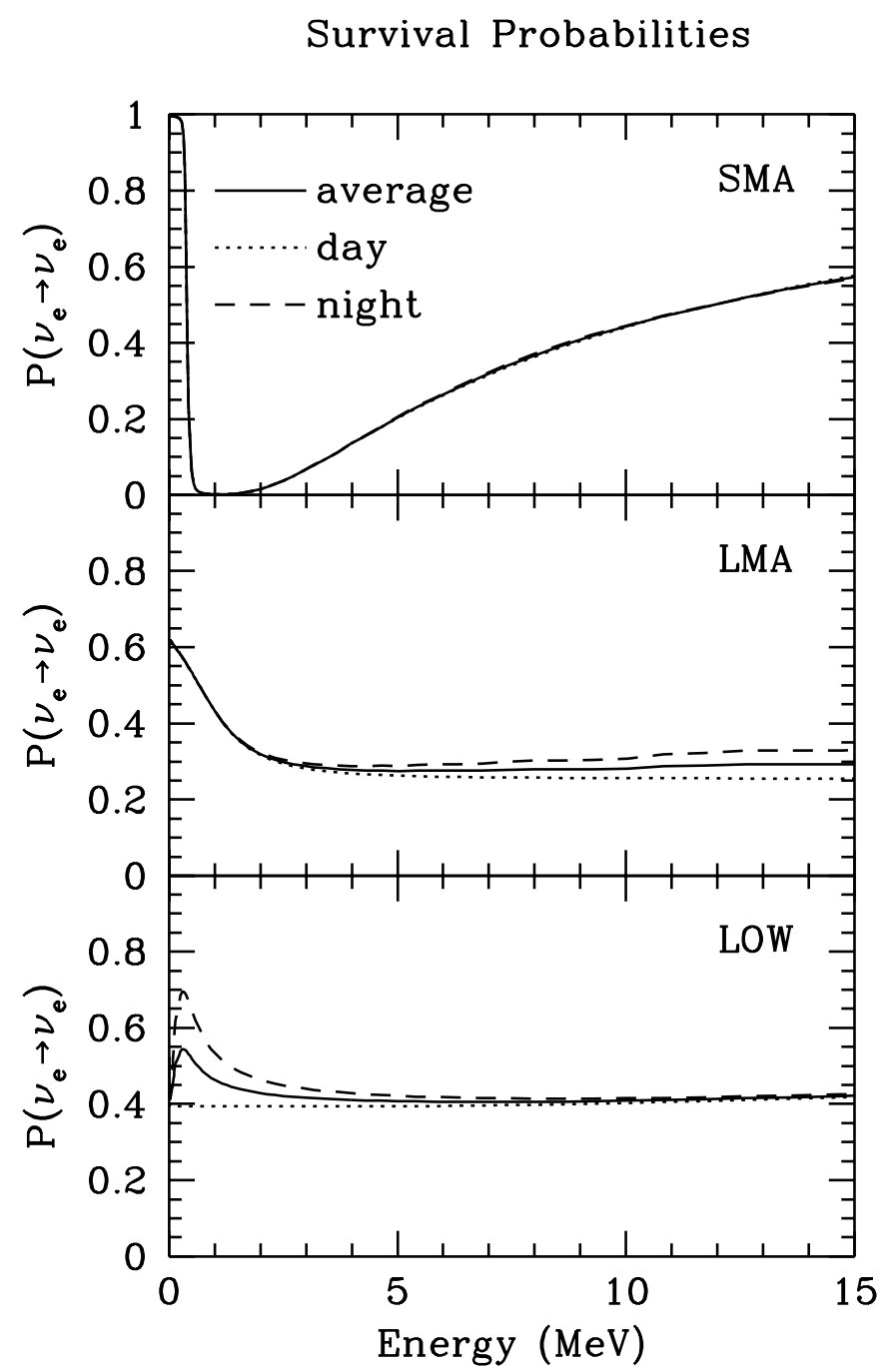

FIG. 9. Survival probabilities for MSW solutions. The figure presents the yearly-averaged survival probabilities for an electron neutrino that is created in the sun to remain an electron neutrino upon arrival at the SuperKamiokande detector. The best-fit MSW solutions including regeneration in the earth are described in Sec. IV. The full line refers to the average survival probabilities computed taking into account regeneration in the earth and the dotted line refers to calculations for the daytime that do not include regeneration. The dashed line includes regeneration at night. There are only slight differences between the computed regeneration probabilities for the detectors located at the positions of Super-Kamiokande, SNO and the Gran Sasso Underground Laboratory (see Ref. 43]). 

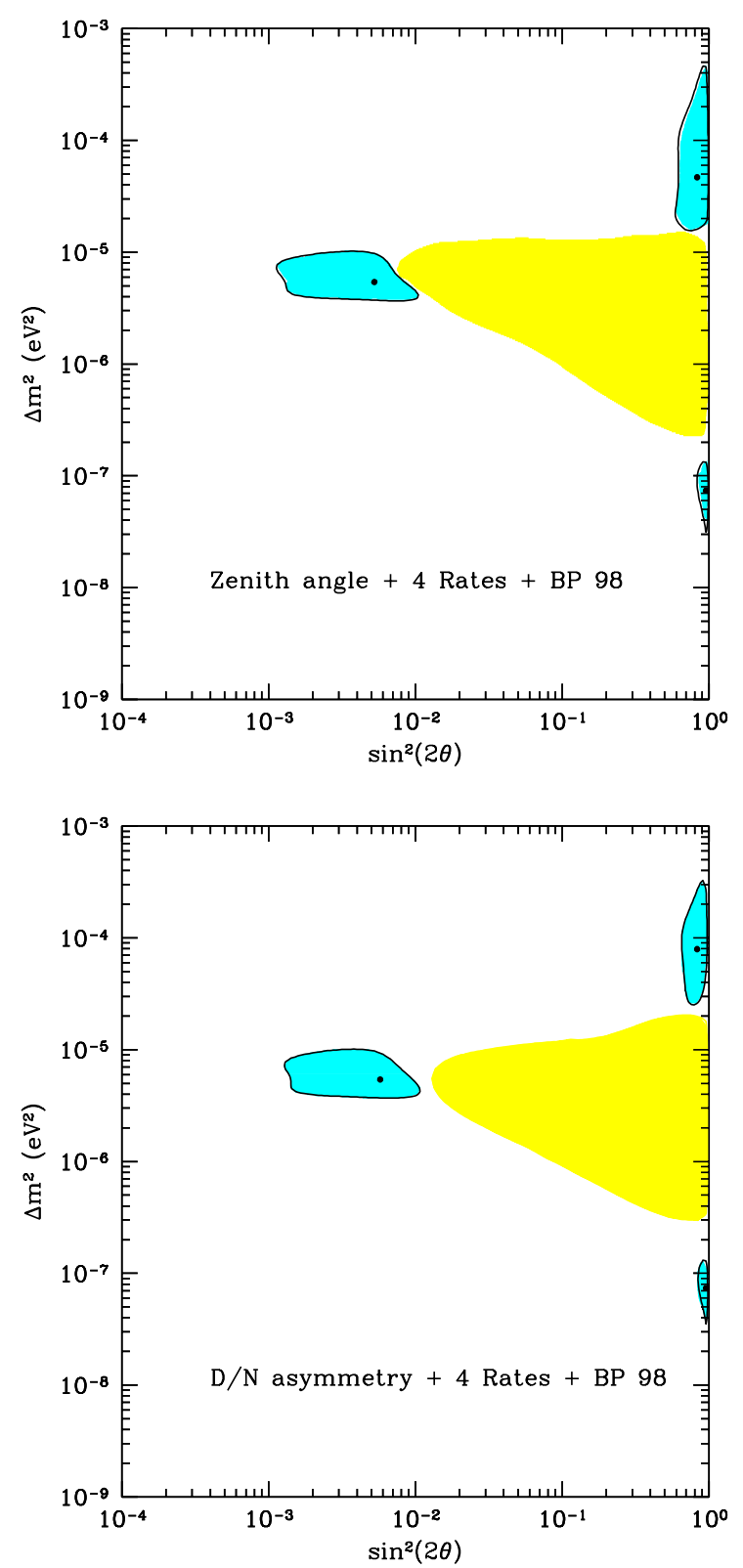

FIG. 10. Angular distribution exclusion. The region that is excluded by the SuperKamiokande zenith-angle distribution or by the SuperKamiokande Day-Night measurement is shown as the light shaded area in the upper and lower panels, respectively. The darker shaded regions in the upper panel are the regions that are allowed by the total measured rates from the chlorine, GALLEX, SAGE, and SuperKamiokande experiments plus the zenith angle distribution measured by SuperKamiokande. The darker shaded regions in the lower panel are allowed by the four measured rates and the SuperKamiokande Day-Night asymmetry. 

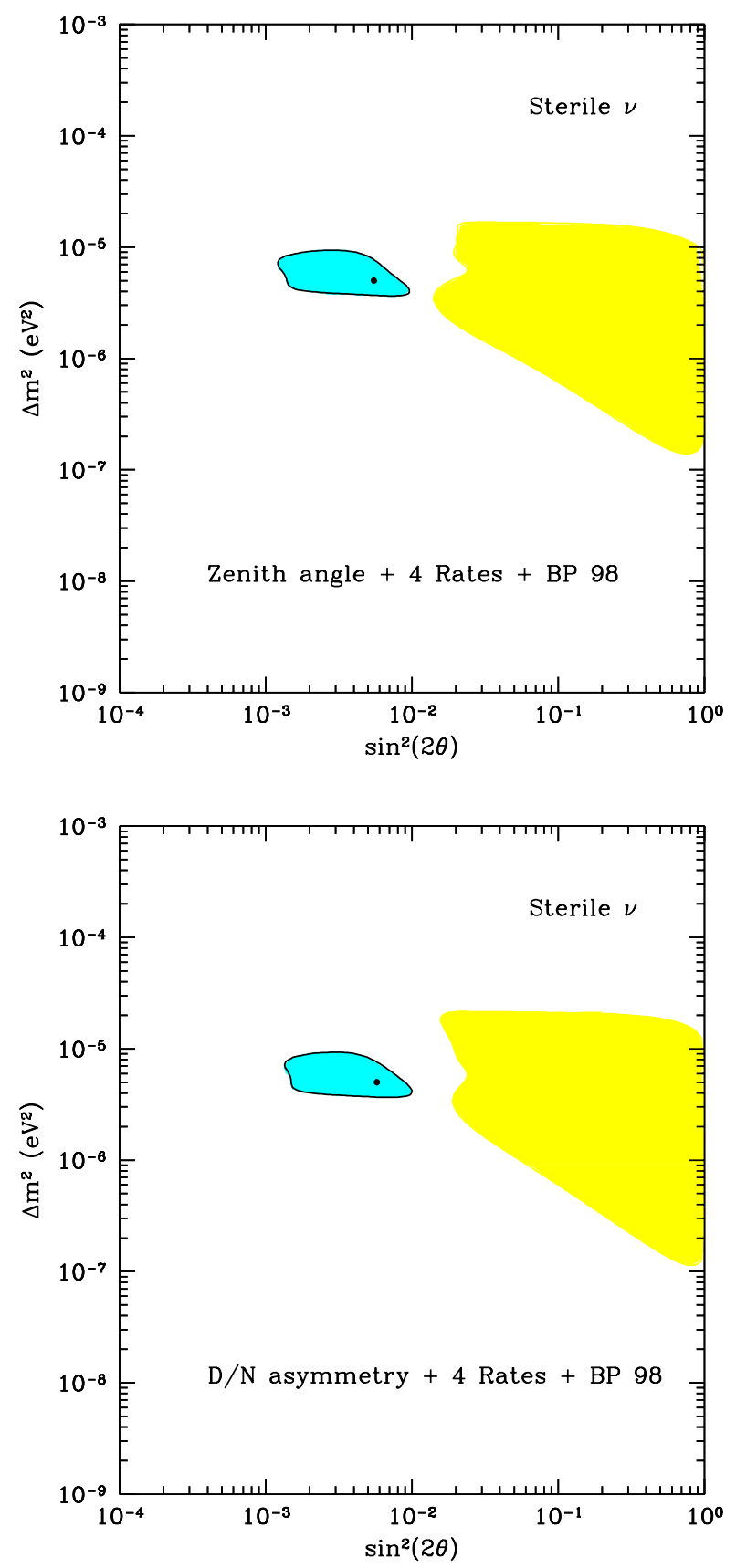

FIG. 11. Angular distribution exclusion for sterile neutrinos. This figure is the same as Fig. 10 except that the present figure refers to oscillations into sterile neutrinos. 


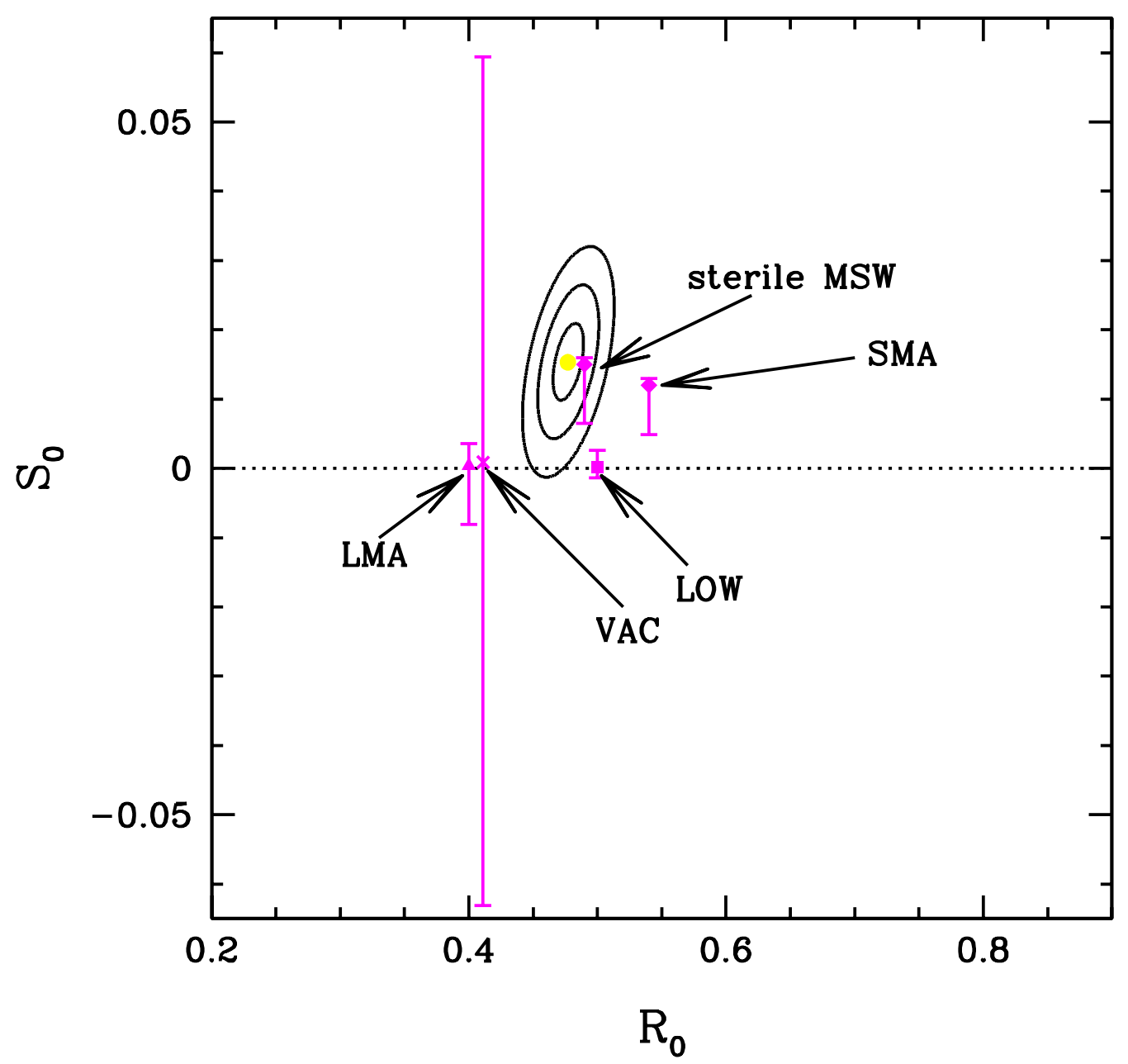

FIG. 12. Deviation from an undistorted energy spectrum. The $1 \sigma, 2 \sigma$, and $3 \sigma$ allowed regions are shown in the figure. The ratio of the observed counting rate as a function of electron recoil energy [10] to the expected undistorted energy spectrum [19] was fit to a linear function of energy, with intercept $R_{0}$ and slope $S_{0}$ (see Eq. 17). The five oscillation solutions discussed in Sec. IV, SMA active and sterile, LMA, LOW, and vacuum oscillations, all provide acceptable fits to the data, although the fits are not particularly good, see text in Sec. V1. 

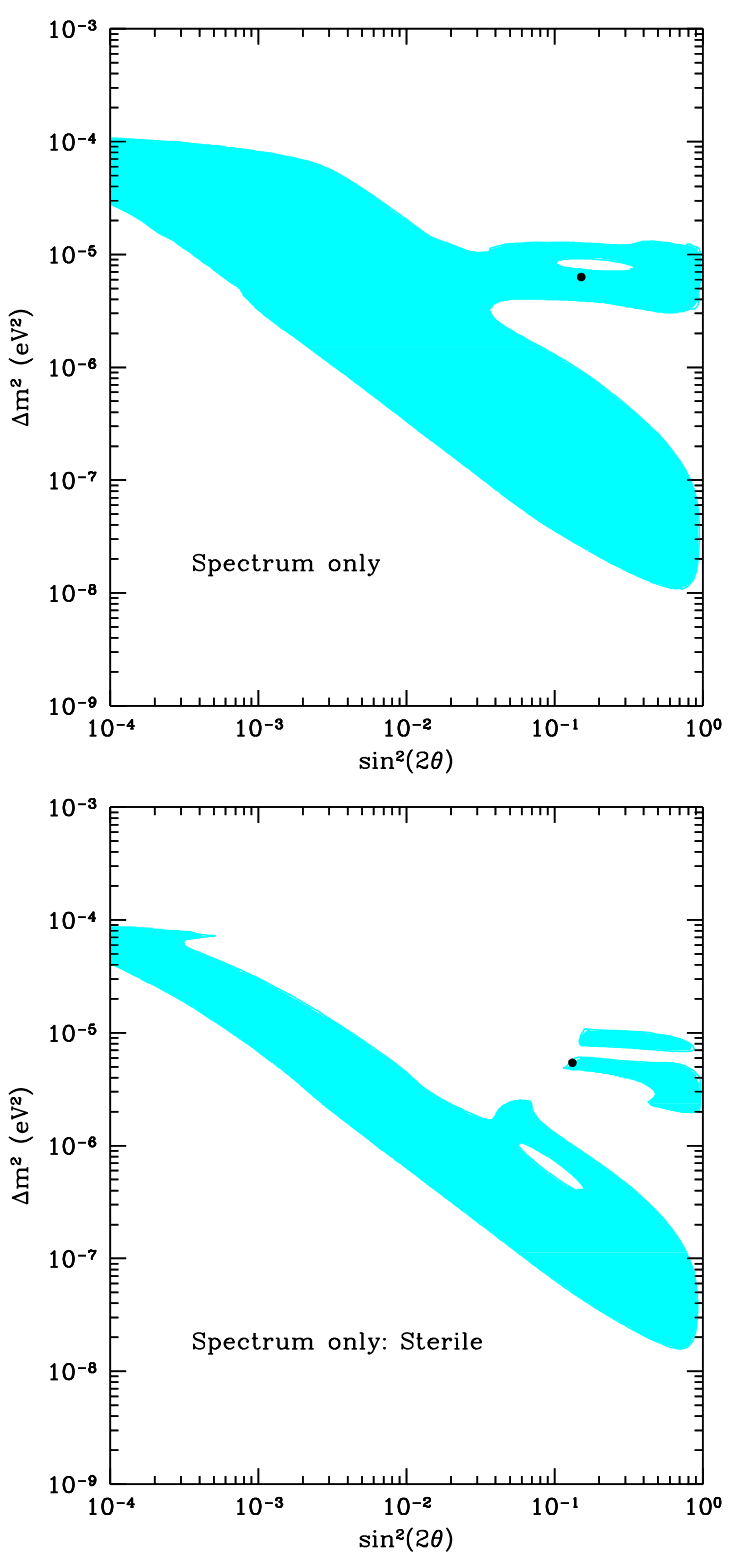

FIG. 13. Spectrum shape: allowed region for MSW oscillations. Figure 13a refers to active neutrinos and Figure 13b refers to sterile neutrinos. Each panel shows the region in MSW solution space that is allowed by the SuperKamiokande [10] measurements of the recoil energy spectrum from the scattering of ${ }^{8} \mathrm{~B}$ neutrinos by electrons. The best-fit solution when only the energy spectrum is considered is shown by dark points in Figure 13a and Figure 13b . It is interesting to compare the regions allowed by the spectral energy distribution with the regions allowed by the total rates, cf. this figure with Fig. 2 and Fig. 4 . 


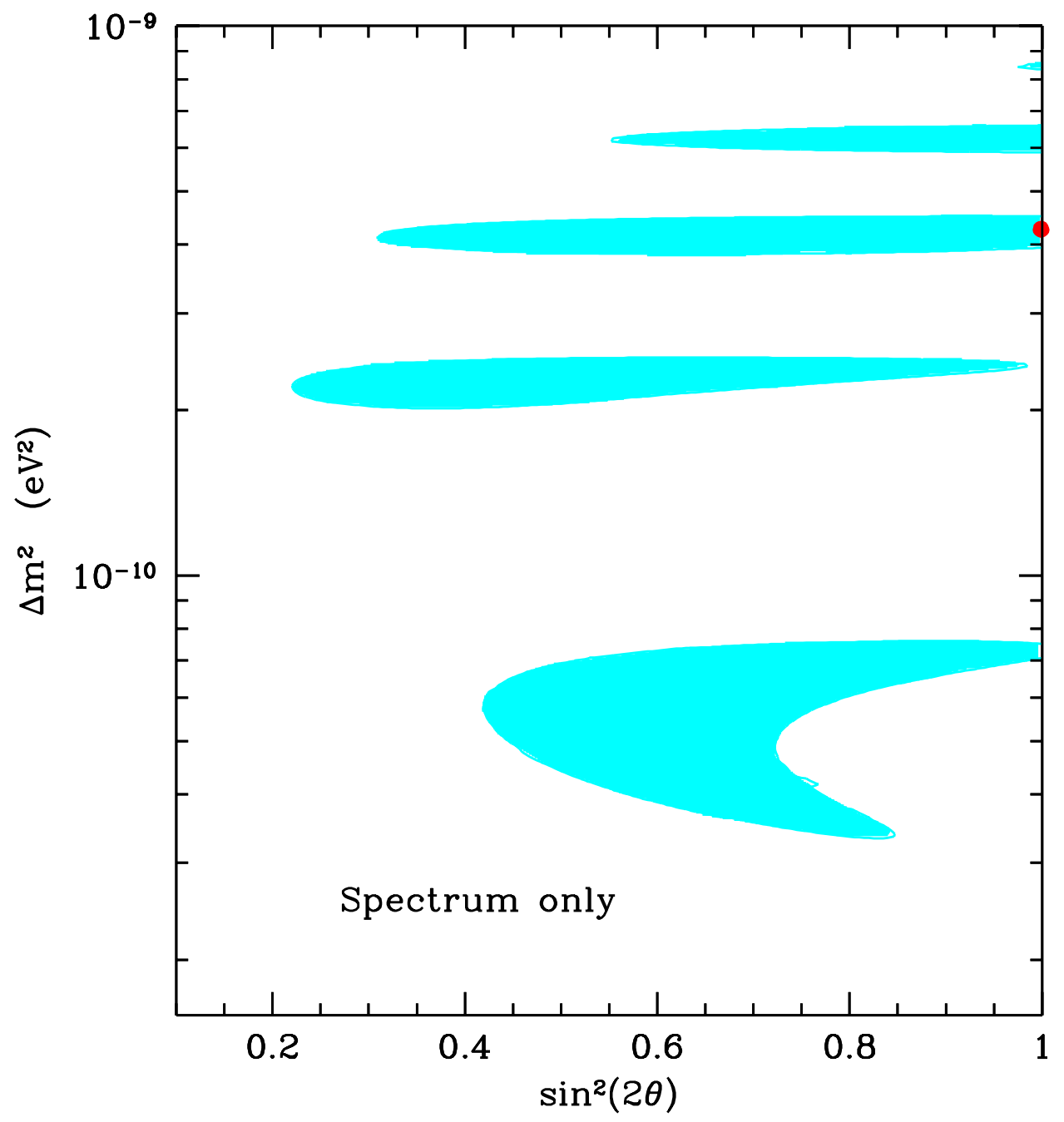

FIG. 14. Spectrum shape: allowed region for vacuum oscillations. The figure shows the allowed region in the parameter space of vacuum oscillations that is permitted by the SuperKamiokande [10] measurements of the recoil electron energy spectrum. cf. Fig. 5 . The dark point shows the best-fit point considering the measured spectrum as the only constraint; the value of $\chi_{\min }^{2}=30.5$. 

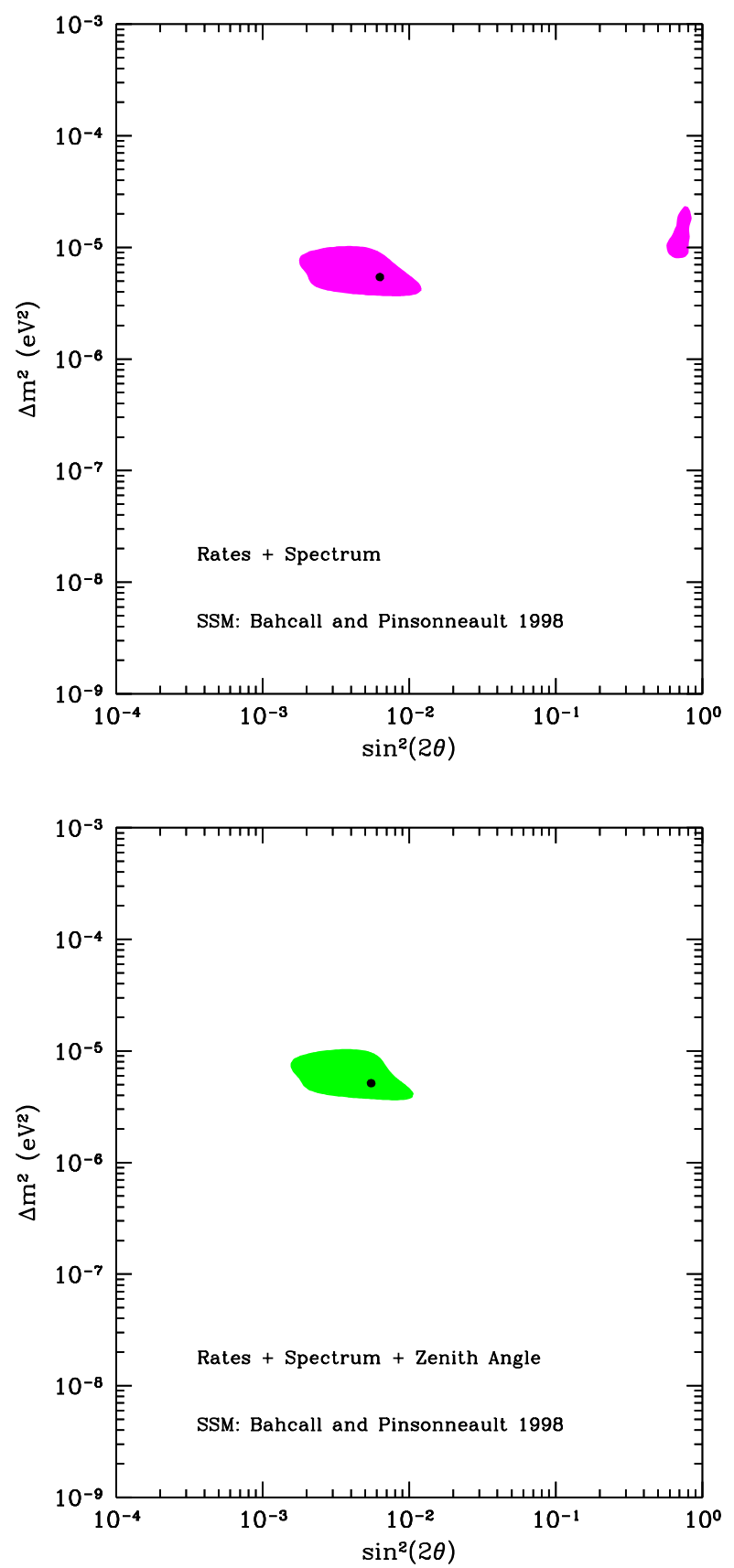

FIG. 15. Global fits: MSW solutions. Figure 15a shows the regions in MSW parameter space that are consistent with the total rates observed in the four solar neutrino experiments (chlorine, SuperKamiokande, GALLEX, and SAGE) and the measured SuperKamiokande electron recoil energy spectrum. Figure $15 \mathrm{~b}$ shows the only allowed region in MSW parameter space that is consistent with the combined constraints from the four measured rates and the electron recoil energy spectrum and zenith angle distribution that are measured by SuperKamiokande. Contours are drawn at $99 \%$ C.L. 


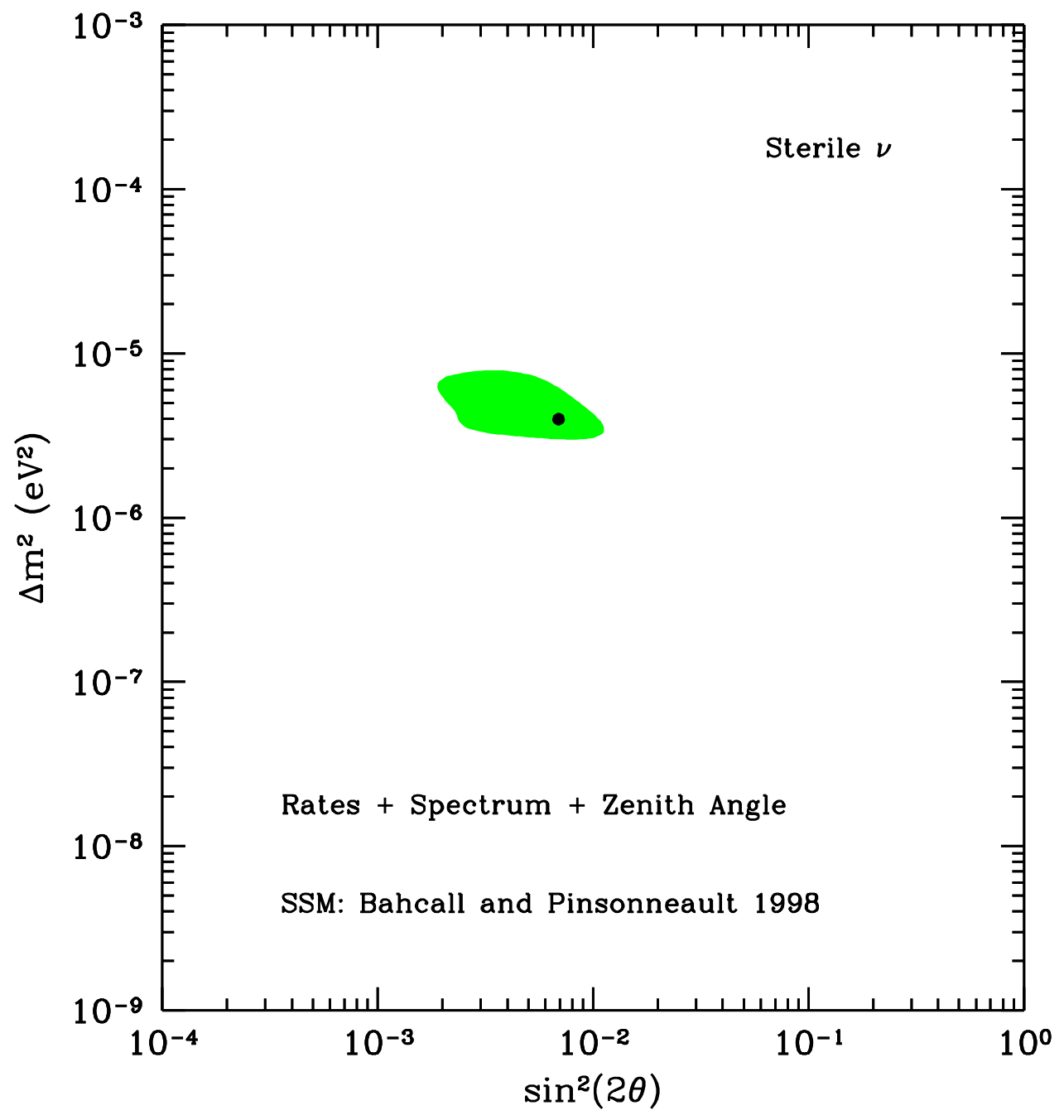

FIG. 16. Global fits: sterile neutrinos. The figure shows the allowed parameter region for MSW oscillations into sterile neutrinos that is consistent with the measured total rates, the zenith-angle distribution, and the recoil electron energy spectrum. Contours are drawn at 99\% C.L. . 


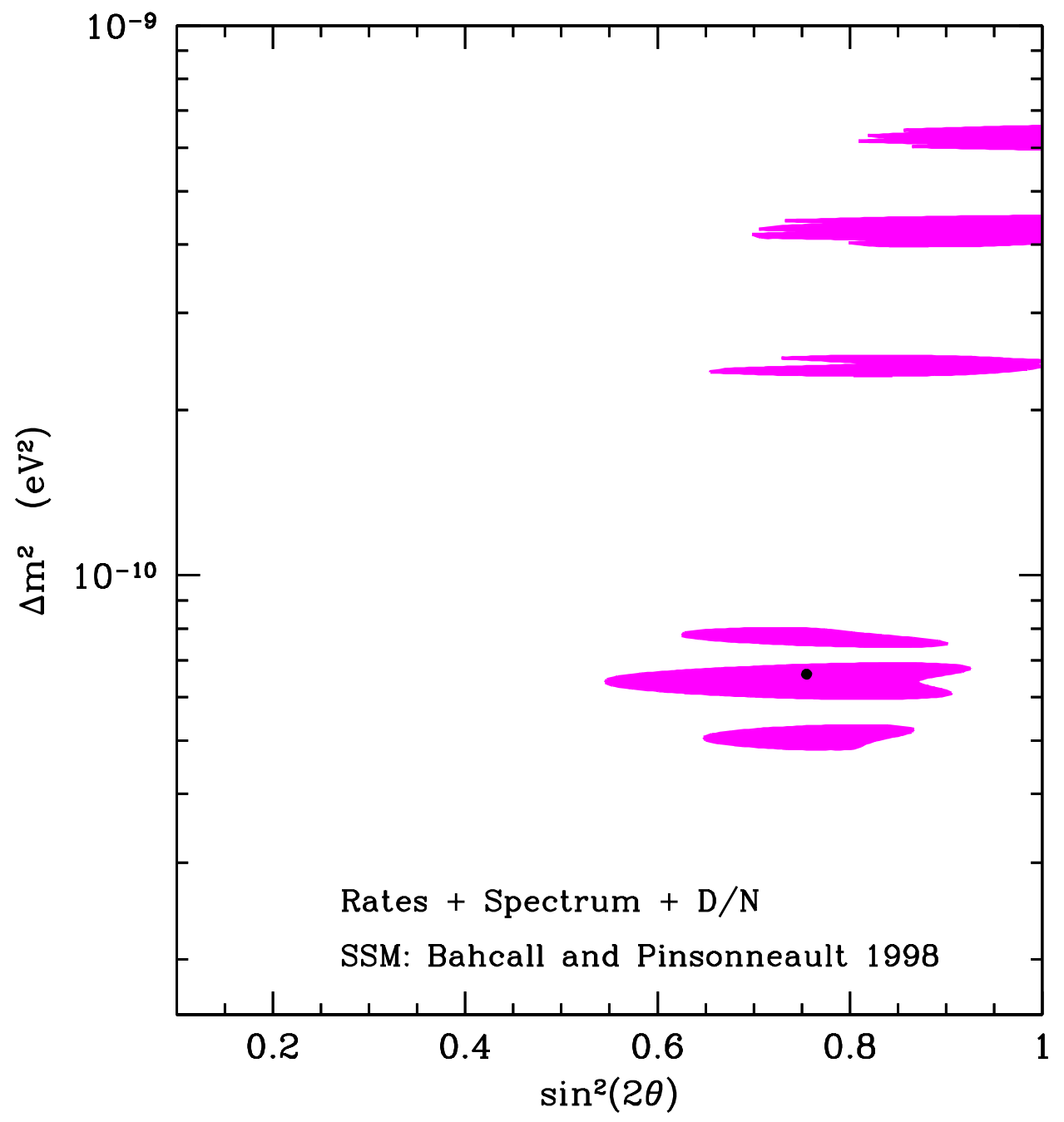

FIG. 17. Global fits: vacuum solutions. The figure shows the allowed parameter region for vacuum oscillations that is consistent with the measured total rates, the recoil electron energy spectrum, and the Day-Night asymmetry. Contours are drawn at $99 \%$ C.L. . 


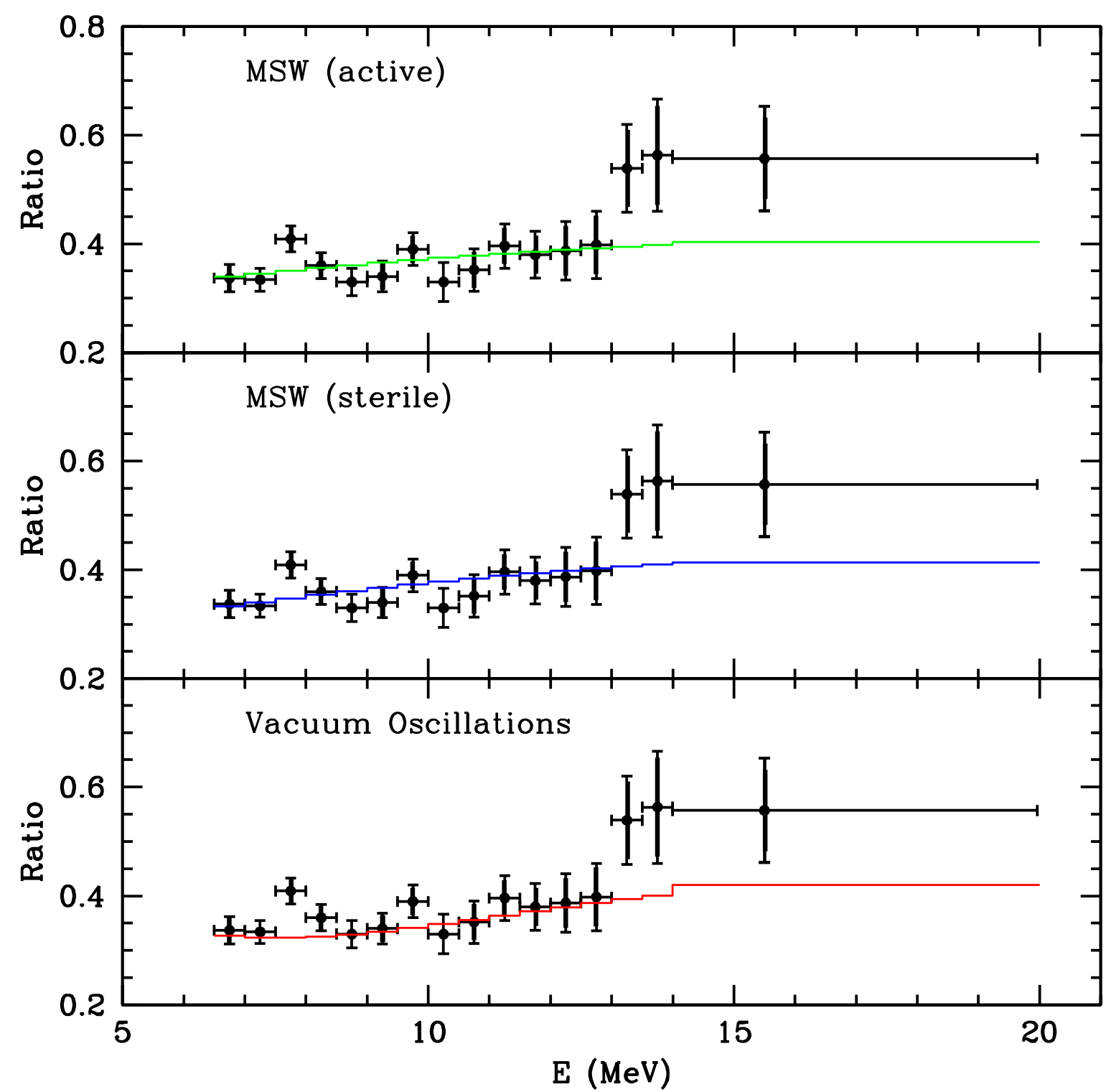

FIG. 18. Global best fits versus measured energy spectrum. The three panels compare the global neutrino oscillation solutions discussed in Section VII versus the electron energy spectrum measured by SuperKamiokande and reported at Neutrino98 [10]. The quantity, Ratio, that is plotted is the ratio of the number of electrons in a given energy bin, $E$, to the number that is calculated using the standard, undistorted ${ }^{8} \mathrm{~B}$ neutrino energy spectrum 19 and electroweak neutrino-electron scattering cross sections with radiative corrections [18]. The no oscillation solution is a horizontal line, which,following the SuperKamiokande collaboration [10], is normalized to the BP95 prediction 27] and lies at Ratio $=0.37$. 